\title{
A constitutive analysis of nonlinear shear flow
}

\author{
Esmaeil Narimissa ${ }^{1,2} \cdot$ Thomas Schweizer $^{3}$ - Manfred H. Wagner ${ }^{4}(\mathbb{D}$
}

Received: 26 January 2020 /Revised: 11 May 2020 / Accepted: 12 May 2020 / Published online: 3 June 2020

(C) The Author(s) 2020

\begin{abstract}
We analyse shear stress and normal stress data obtained by cone-partitioned-plate (CPP) shear rheometry in recent years. The data sets of Schweizer et al. (Rheol. Acta 47, 943-957, 2008) and Costanzo et al. (Macromolecules 49, 3925-3935, 2016; \& Fluids 4, $28,2019)$ on nearly monodisperse polystyrene melts and solutions are considered to be among the most reliable shear data available. The Doi-Edwards independent alignment (DEIA) model (J. Chem. Soc., Faraday Transactions 2: Molecular and Chemical Physics 74, 1802-1832, 1978a,b) allows for quantitative description of the steady-state values of shear viscosity $\eta(\dot{\gamma})$ and first normal stress coefficient $\psi_{1}(\dot{\gamma})$, while it underpredicts the stress overshoot of the stress growth coefficient of the shear stress, $\eta^{+}(t)$, and fails in predicting a stress overshoot of the stress growth coefficient of first normal stress difference, $\psi_{1}^{+}(t)$. On the other hand, the extended interchain pressure (EIP) model (J. Rheol. 64, 95-110, 2020) provides an excellent prediction of the stress overshoots of both shear stress and first normal stress difference, while overpredicting the steady-state shear viscosity and the first normal stress coefficient. We demonstrate that the shear stress overshoot is the result of a combination of orientational stress overshoot and stretch overshoot, while the normal stress overshoot depends solely on the overshoot of the stretch. Based on these considerations, we propose a novel constitutive approach consisting of a combination of the DEIA and the EIP model, and predictions of this approach are found to be in quantitative agreement with the data sets of Schweizer et al. and Costanzo et al. within experimental accuracy.
\end{abstract}

Keywords Shear rheometry $\cdot$ Cone-partitioned-plate $\cdot$ Doi-Edwards model $\cdot$ MSF model $\cdot$ Shear stress overshoot $\cdot$ Normal stress overshoot $\cdot$ Polystyrene melts and solutions

\section{Introduction}

The molecular structure of polymeric melts and solutions plays the primary role in determining their rheological properties; hence, the constitutive equations based on mesoscopic molecular modelling are significant in rheological modelling. In particular, the tube-based models have been able to reflect

Manfred H. Wagner

manfred.wagner@tu-berlin.de

1 Dept. of Chemical Engineering, Technion-Israel Institute of Technology (IIT), Technion City, 32000 Haifa, Israel

2 Dept. of Chemical Engineering, Guangdong Technion-Israel Institute of Technology (GTIIT), Shantou 515063, China

3 Department of Materials, Polymer Physics, ETH Zürich, CH-8093 Zürich, Switzerland

4 Polymer Engineering/Polymer Physics, Berlin Institute of Technology (TU Berlin), Ernst-Reuter-Platz 1, 10587 Berlin, Germany fundamental molecular aspects of polymers through averaging the effect of neighbouring chains on the test chain via a mean-field approach. The tube model of Doi-Edwards (DE) (Doi and Edwards 1978b; Doi and Edwards 1978a; Doi and Edwards 1979; Doi and Edwards 1986) was originally developed for monodisperse linear polymers based on the idea of the reptating motion of a linear entangled chain with molar mass $M$ in a tube with equilibrium contour length of $L_{0}=N a_{0}$, where $N$ is the number of primitive path steps connecting two consecutive entanglements, and $a_{0}$ is the tube diameter. The tube concept is based on the hypothetical view of a test chain laterally confined in a mesh of constraints created by the surrounding chains having the same molar mass as the test chain. Upon deformation, the recovery of the contour length of the primitive chain (i.e. relaxation) occurs due to the reptation (chain diffusion) and retraction mechanisms. As originally described by de Gennes (De Gennes 1971), during reptation, the test chain escapes from the tube by sliding back and forth within the tube until completely moves its mass out of the original tube. The relaxation time associated with the reptation 
mechanism is the disengagement (or reptation) time $\tau_{d} \propto M^{3}$. During the fast retraction mechanism, the contour length is recovered after a Rouse relaxation process governed by the Rouse time $\tau_{R} \propto M^{2}$. Based on the independent alignment approximation (IA) of DE theory (DEIA), the tube segments are assumed to be aligned independently in the field of flow where stress after rapid equilibration of chain stretch is created by affine rotation of tube segments (see Fig. 1 of Narimissa and Wagner 2019). The tube model with and without the IA assumption generates similar predictions for uniaxial
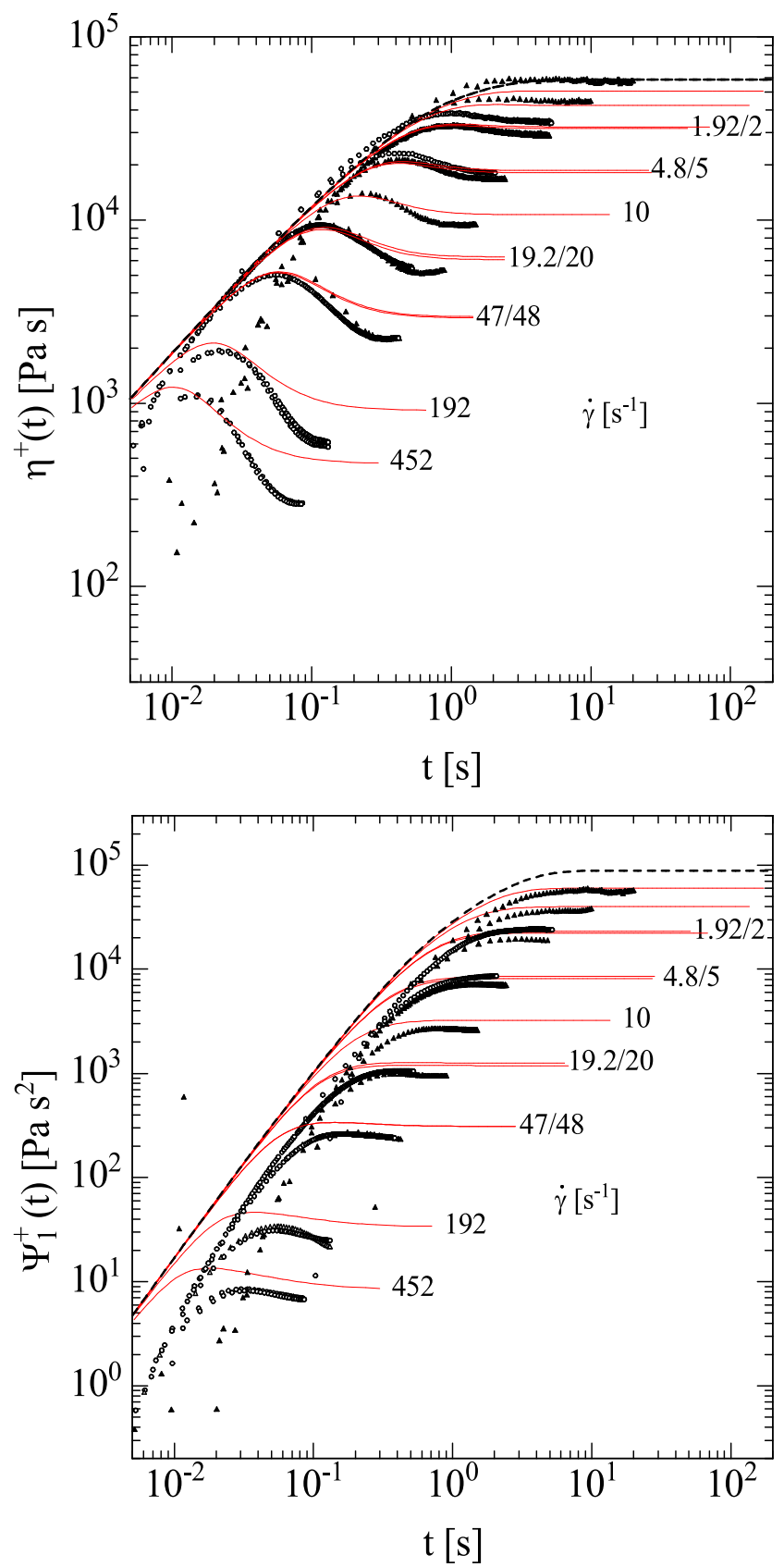

Fig. 1 Comparison between the predictions (red lines) of the EIP model (Eqs. (13) and (14)) and the shear stress growth coefficient $\eta^{+}(t)$, and the first normal stress growth coefficient $\psi_{1}^{+}(t)$ of PS-206k at $180{ }^{\circ} \mathrm{C}$ extensional flow, yet, the predictions of biaxial flows and the second normal stress difference in shear flow are different.

Doi and Edwards showed (Doi and Edwards 1978a, see e.g. their Fig. 6 for the damping function) that their tube model is in nearly quantitative agreement with the stress relaxation data after step shear strain of Osaki et al. (Fukuda et al. 1975; Osaki et al. 1975; Osaki et al. 1976). However, the tube model cannot predict the occurrence of overshoot in the first normal stress function in shear, and it does not predict chain stretch in elongational flow leading to the extension-thinning exponent of -1 as opposed to the experimentally observed exponent of approximately -0.5 in polymer melts (Narimissa and Wagner 2019). Several modifications have been made to the original tube model to address its shortcomings for modelling the linear and nonlinear viscoelastic rheology of polymers. In the linear-viscoelastic regime (LVE), contour length fluctuations (CLF) (Doi 1981) and constraint release (CR) (Daoud and De Gennes 1979) were introduced to the DE model to address the experimentally observed disagreements between the LVE behaviours of linear entangled polymers and the theory, specifically the 3.4 power scaling of the zero-shear viscosity. In the nonlinear-viscoelastic regime, the predictions of the DE model are only in qualitative agreement with the flow behaviour of monodisperse linear entangled polymers subjected to shear deformation, and the model does not predict the strain hardening in the start-up of the extensional flows (Wagner et al. 2005). We note that the DEIA single integral equation is of the Kaye-BKZ type (Bernstein et al. 1963) and as shown by Samurkas et al. (1989) (see also Laun and Schuch 1989), the Kaye-BKZ and Wagner (Wagner 1977) single integral equations cannot simultaneously describe both strain softening in shear and extreme strain hardening in planar extension using a damping function obtained from one of these flows. These discrepancies between model and experimental evidence have led to the introduction of convective constraint release (CCR) (Ianniruberto and Marrucci 1996) (to avoid the excessive shear thinning of the DE model) as well as chain stretch mechanisms (Doi and Edwards 1986; Marrucci and Grizzuti 1988) in predominantly differential approximations and variations of the tube model with pre-averaged chain stretch (see Review (Narimissa and Wagner 2019) for more details).

Regarding the tube model-based constitutive equations without pre-averaged chain stretch (Narimissa and Wagner 2019) for polydisperse melts, the molecular stress function (MSF) model (Wagner et al. 2001; Wagner and Schaeffer 1992b; Wagner and Schaeffer 1993; Wagner and Schaeffer 1994; Wagner et al. 2003) and the hierarchical multi-mode molecular stress function (HMMSF) model (Narimissa et al. 2015; Narimissa et al. 2016; Narimissa and Wagner 2016a; Narimissa and Wagner 2016b; Narimissa and Wagner 2016; Narimissa and Wagner 2018) were developed based on the strain-dependent tube diameter theory of Marrucci and de Cindio (1980) (Marrucci and de Cindio 1980). In the MSF 
and HMMSF models, the constraint release (CR) only affects the chain stretch and tube diameter, which is in contrast to the differential tube-based models with pre-averaged chain stretch (refer to section B of Narimissa and Wagner 2019) where CCR is taken primarily to be a mechanism for relaxing tube orientation. Furthermore, the validity of the CR only in shear deformation was well depicted by Bastian (Bastian 2001) (see Fig. 3 in Narimissa and Wagner 2016) showing that during shear flow, the affine change in tube cross-sectional area continues, and the topological constraints above and below the chain are released; hence, tube diameter returns to its equilibrium value $a_{0}$. This contrasts with the extensional flow where the CR effect is compensated by the advection of neighbouring topological constraints, hence, a minimum tube diameter and maximum stretch are achieved.

To explain the difference between the extensional flow behaviours of melts and solutions, Ianniruberto (2015) and Park and Ianniruberto (2017) proposed a flow-induced monomeric friction $\zeta$ reduction mechanism which is only effective in melts. Due to $\zeta$ reduction, the relaxation times become time-dependent during fast deformations. However, recent molecular dynamics (MD) simulations of stress relaxation after elongational flow of a highly oriented entangled polymer melt using a Kremer-Grest bead spring model failed to find direct evidence of monomeric friction reduction (O'Connor et al. 2019). On the other hand, Costanzo et al. (2016) showed the insignificance of the CCR and $\zeta$ reduction mechanisms during the fast shear deformation of monodisperse polystyrene (PS) melts and solutions. They claimed that in order to capture the occurrence of undershoot during fast shear flows, an essentially phenomenological concept generated from molecular dynamic simulations (Nafar Sefiddashti et al. 2014), i.e. tumbling, must be considered. In addition, inclusion of the tumbling function in the stretch evolution equation (i.e. tumbling-induced stretch reduction) also allowed predicting the steady-state values of the first and second normal stress difference of PS solutions (Costanzo et al. 2018).

Recently, we developed an extended interchain pressure (EIP) model for monodisperse polystyrene melts and concentrated polystyrene solutions in oligomeric styrene (Narimissa et al. 2020). The EIP model explains the effect of molar mass of the solvent on the extension-thickening behaviour of solutions, and with only 2 equations (i.e. stress equation and stretch evolution equation) and without any free nonlinearviscoelastic parameter, the EIP model predicts that at low Weissenberg numbers $W i_{R}=\dot{\varepsilon} \tau_{R}<1$, melts and solutions show extension-thinning, while at $W i_{R} \cong 1$ solutions switch to extension-thickening or show a more or less constant steady-state elongational viscosity, and melts continue with extension-thinning behaviour with a scaling of $\eta_{E} \propto W i_{R}^{-0.5}$. The EIP model explains quantitatively the effects of molar mass of solvent and of polymer concentration on the elongational viscosity in the investigated concentration range from 10 to $100 \%$ (melt), based solely on the linear-viscoelastic characterization and in agreement with available experimental evidence (Acharya et al. 2008; Bach et al. 2003; Bhattacharjee et al. 2002; Huang et al. 2013a; Huang et al. 2015; Huang et al. 2013b). However, the applicability of the EIP model to shear flow has not been tested.

On the experimental side, nonlinear shear rheometry with cone-plate and plate-plate tools of polymer liquids at large Weissenberg numbers is affected by the formation of flow instabilities such as shear banding, wall slip and most importantly, the edge fracture (Costanzo et al. 2016; Tanner and Keentok 1983), while normal stress difference measurements are hampered by limited compliance of cone-plate rheometers (Meissner et al. 1989; Schweizer et al. 2008). For example, Osaki et al. (2000) investigated the overshoot and detected also undershoot in both growth coefficients of shear stress and first normal stress in a series of PS solutions up to high shear rates. They noticed that at shear rates $\dot{\gamma}>50 \mathrm{~s}^{-1}$, both stresses continue increasing and steady state was not attainable. Later, Auhl et al. (2008) reported that in monodisperse polyisoprene melts, the shear stress growth coefficient demonstrates both overshoot and undershoot only at $W i=14$, while overshoot in the first normal stress difference occurs much later in time when no undershoot was detected. They noted that experimental shear data for high molecular mass polymers at large strains must be treated with caution due to instabilities such as edge fracture and (possibly) wall slip. Menezes and Graessley (1982) demonstrated that by incorporating an equilibration time in a simplified (Wagner type) BKZ constitutive equation (Wagner 1977), it is possible to predict overshoot as well as undershoot with damping functions obtained from step-strain relaxation data. Tanner and Keentok (1983) and Keentok and Xue (1999) conducted comprehensive studies on the edge fracture phenomenon and showed that this flow instability effect is governed by the second normal stress difference of the material. In a series of publications on shear banding, Wang and co-workers (Boukany and Wang 2007; Ravindranath et al. 2008; Tapadia and Wang 2006) claimed that entangled polymeric systems fail to maintain uniform deformation when sheared on time scales faster than their terminal relaxation time and no stable shear flow is possible after the shear stress overshoot. Schweizer et al. (2008) suggested that edge fracture might initiate shear banding. Following Wang and co-workers' findings (Boukany and Wang 2007, Ravindranath et al. 2008, Tapadia and Wang 2006), Moorcroft and Fielding (2013) applied the Rolie-Poly model to show that in start-up shear flows, shear banding (i.e. purely elastic flow instability) occurs as the result of stress overshoot. They also proposed a stability criterion for startup shear flow when the flow becomes unstable to banding. 
Meissner et al. (1989) were the first searching for an experimental method to deal with the onset of flow instabilities in the form of material separation (i.e. edge fracture) at the rim of the sample in cone-plate (CP) rheometry, and they introduced the cone-partitioned-plate (CPP) geometry by partitioning the plate of the CP system into an inner disk connected with the measuring transducer, and an outer ring fixed to the instrument frame. Following Meissner et al. (1989), significant progress in rotational shear rheometry was made by the development of CPP tools (Schweizer and Schmidheiny 2013; Schweizer and Stöckli 2008; Schweizer et al. 2004), which alleviate the negative impacts of the edge fracture on torque and normal stress measurements by expanding the sample volume, hence postponing its effect on the (inner) measurement partition (see (Costanzo et al. 2016) for more details). Snijkers and Vlassopoulos (2011) showed that the edge fracture in standard rotational rheometers (cone-and-plate or plateplate geometries) plays a catastrophic role in the bulk measurement of the shear rheology of the fluid. Furthermore, they summarized the earlier results obtained by CP rheometry (before the introduction of cone-partitioned-plate geometry) and clearly showed that no homogeneous shear flow can be achieved at high Weissenberg numbers (Wi). Thus, all earlier reports of steady-state shear viscosity at high $W i$ including shear stress undershoot followed by steady-state before the introduction of CPP rheometry are questionable.

Through the introduction of CPP, higher Wi measurements became possible. In CPP rheometry, it is assumed that effects such as edge fracture and/or shear banding are limited to the outer partition, and shear flow stays homogeneous in the innermost partition. Although no proof of such assumption has been presented so far and it remains unclear at which $W i$ the limits of CPP rheometry are reached, enhanced reliability of $\mathrm{CPP}$ measurements at higher $\mathrm{Wi}$ as compared to CP was reported (Snijkers and Vlassopoulos 2011). The shear data of Costanzo et al. 2016 and Stephanou et al. (2017) showed that at high $W i$, the overshoot in stress growth coefficient is followed by a shear stress minimum (i.e. undershoot) before reaching the steady state. Snijkers and Vlassopoulos (2011) did not mention undershoot, although a slight undershoot is seen in their shear data. Whether the undershoot observed at high $W i$ with CPP is a true phenomenon or caused by e.g. shear banding penetrating into the inner partition is still an open question. Costanzo et al. (2018) did not detect any undershoot in transient first normal stress difference of PS solutions, and they (Costanzo et al. 2016; Costanzo et al. 2018) related the undershoot in the shear stress growth coefficient to the molecular tumbling relaxation mechanism within the entangled polymer chains (Nafar Sefiddashti et al. 2015) and proposed a phenological modification to a tube-based model to account for molecular tumbling. Stephanou et al. (2017) used the tumbling-snake model to explain overshoot and undershoot of a concentrated PS solution. However, the molecular tumbling relaxation mechanism was experimentally observed only in dilute DNA solutions as summarized by Smith et al. (1999) for concentrations $10^{4}-10^{5}$ times lower than the concentration when coiled molecules begin to overlap. Therefore, the credibility of molecular tumbling theory in concentrated polymer solutions and polymer melts is highly disputed, and the citation of this theoretical phenomenon has been primarily reliant on molecular dynamic simulations (e.g. Nafar Sefiddashti et al. 2015, 2017, 2019a,b and Masubuchi et al. 2018) for such polymeric systems. However, the undershoot effect seen in these simulations is much less pronounced than what is observed experimentally, and the most recent molecular dynamic simulations (Kremer-Grest chains) study conducted by Anwar and Graham (2019) did not show the occurrence of undershoot in either shear stress growth coefficient or the first normal stress coefficient of entangled polymers. Therefore, due to the ambiguity of the nature of the experimentally observed shear stress undershoot as well as the disputed numerical simulation basis of the molecular tumbling theory, we refrain from modelling the undershoot in this study; yet, we will concentrate on a quantitative description of the shear stress and first normal stress overshoots. We also refrain from considering the second normal stress function $N_{2}$, which depends on the curvature at the rim of the sample in cone-plate rheometry and which mirrors the undefined and/or unstable free surface situation at the rim (see e.g. Schweizer et al. 2008; Venerus 2007).

In this study, we analyse shear stress and normal stress data of well-defined polystyrene (PS) melts and solutions obtained by CPP shear rheometry in recent years. The data sets analysed are among the most reliable shear data available. After a short introduction of the DEIA and EIP models, we start our analysis by applying the EIP model to the shear data of a PS melt investigated by Schweizer et al. (2008). Comparison of data and model demonstrate the need for a modification of the model for shear flow. Introduction of CR was found to be insufficient. An innovating approach to modelling rotational flows is presented, and predictions are compared with the data set of Schweizer et al. (2008) as well as PS melts and solutions data sets of Costanzo et al. (2016) and Costanzo et al. (2019).

\section{Experimental data and LVE characterization}

\section{Materials}

The shear rheological modellings of monodisperse polymer melts and solutions were conducted on a series of PS melts and PS/oligomeric styrene (OS) solutions with different weight-average molar masses and weight fractions previously investigated by Schweizer et al. (2008) and Costanzo et al. (2016) and Costanzo et al. (2019). Table 1 
Table 1 Components, weight percentage, molar mass, polydispersity index, measuring temperature, Rouse time, disengagement time, zeroshear viscosity, glass transition temperature, and testing environment of polystyrene melts and solutions, and oligomeric styrenes. Data summarized from Costanzo et al. (2016), Costanzo et al. (2019) and Schweizer et al. (2008)

\begin{tabular}{lccccccc}
\hline Sample name & $\varphi[\mathrm{wt} . \%$ of PS $]$ & $M[\mathrm{~g} / \mathrm{mol}]$ & PDI & $T\left[{ }^{\circ} \mathrm{C}\right]$ & $T_{g}\left[{ }^{\circ} \mathrm{C}\right]$ & Rheometer & Ref. \\
\hline PS-206k & 100 & 206,000 & 1.06 & 180 & - & MTR 25 & Schweizeret al. (2008) \\
PS-133k & 100 & 103,000 & 1.01 & 160 & - & ARES & Costanzo et al. (2016) \\
PS-185k & 100 & 185,000 & 1.03 & 160 & - & ARES & Costanzo et al. (2016) \\
PS-200k & 100 & 202,100 & 1.03 & 160 & - & ARES & Costanzo et al. (2019) \\
PS-285/2k-65 & 64.9 & 285,000 & 1.09 & 150 & - & ARES & Costanzo et al. (2016) \\
PS-285/2k-47 & 46.7 & 285,000 & 1.09 & 140 & - & ARES & Costanzo et al. (2016) \\
PS-545/1k-52 & 52 & 545,000 & 1.12 & 130 & 54.0 & ARES & Costanzo (2020) \\
$1 \mathrm{k}$ & 0 & 972 & 1.12 & - & - & - & - \\
$2 \mathrm{k}$ & 0 & 1920 & 1.08 & - & 60.5 & - & -
\end{tabular}

displays the characterization of the samples including their weight percentage, molar mass, polydispersity index (PDI), testing temperature $(T)$, glass transition temperature $(T g)$, and their testing environment.

\section{Rheological measurements}

Schweizer et al. (2008) used an MTR 25 rheometer to investigate the linear and nonlinear shear flow behaviours of PS 206k. MTR 25 allows direct measurement of first and second normal stresses from a single sample and provides high resolution at high deformation rates (Schweizer et al. 2008). Costanzo et al. (2016) and Costanzo et al. (2019) used an ARES rheometer equipped with a cone-partitioned-plate (CPP) fixture with 6- and 4-mm inner plate radii to analyse linear and nonlinear shear flow properties of PS melts (PS133k, PS-185k, PS-200k) and PS/OS solutions (PS-285/2k65, PS-285/2k-47, PS-545/1k-52).

The linear-viscoelastic characterization in the experimental frequency window was obtained via a parsimonious relaxation spectrum:

$G(t)=\sum_{i=1} g_{i} \exp \left(-t / \tau_{i}\right)$

The partial moduli $g_{i}$ and relaxation times $\tau_{i}$ of the samples as computed by the IRIS software (Winter and Mours 2006) are presented in Tables 2 and 3.

The Rouse stretch relaxation time of polymeric systems $\tau_{R}$ as a function of the testing temperature $T$ (Kelvin) is calculated according to Isaki et al. (2003), Menezes and Graessley (1982), Osaki et al. (1982) and Takahashi et al. (1993):

$\tau_{R}=\frac{12 M \eta_{0}}{\pi^{2} \rho R T \varphi}\left(\frac{M_{c m}}{M \varphi}\right)^{2.4}$
$M_{c m}$ denotes the critical molar mass in the melt state and was taken as $M_{c m}=35000 \mathrm{~g} / \mathrm{mol}$ (Ferry 1980; Luap et al. 2005; Wagner 2014). $\rho$ is the density of PS melt at the testing temperature, $R$ is the gas constant, and $\varphi$ is the volume fraction of polymer in the solution with $\varphi=1$ specifying the melt. (We note that volume and weight fraction of PS dissolved in oligomeric styrene are nearly identical as the densities of PS and OS are nearly identical.)

With $M$ being the molar mass of the polymer, the number of entanglements per chain, $Z$, is obtained,

$Z=\frac{M}{M_{e}}$

The relation between the entanglement molar mass of solution, $M_{e}$, and melt, $M_{e m}$, is given by

$M_{e}=M_{e m} \varphi^{-\alpha}$

The value of the dilution exponent $\alpha$ is model dependent $(1<\alpha<4 / 3$ ) (Auhl et al. 2009; Bhattacharjee et al. 2002; Larson 2001; Park and Larson 2003; Watanabe et al. 2004), and a value of $\alpha=1$ is taken here (Huang et al. 2013b). The number of Kuhn segments between entanglements is $N_{e}=M_{e} /$ $M_{0}$, where $M_{0}$ is the molar mass of the Kuhn segment. For polystyrene melt, we take $M_{e m}=13300 \mathrm{~g} / \mathrm{mol}$ [6] and $M_{0}=$ $610 \mathrm{~g} / \mathrm{mol}$ (Huang et al. 2013a), and the value of $M_{0}$ for polystyrene melts and solutions is assumed to be the same (Huang et al. 2013b).

According to the DE model, the disengagement (or reptation) time $\tau_{d}$ and the zero-shear viscosity $\eta_{0}$ are given by (Dealy et al. 2018),

$$
\begin{aligned}
& \tau_{d}=3 Z^{3} \tau_{e}=3 Z \tau_{R} \\
& \eta_{0}=\frac{\pi^{2}}{12} G_{N}^{0} \tau_{d}
\end{aligned}
$$


Table 2 Parsimonious spectra of PS melt samples by IRIS software (Winter and Mours 2006)

\begin{tabular}{|c|c|c|c|c|c|c|c|c|}
\hline \multirow[t]{2}{*}{ Parsimonious spectrum, $i$} & \multicolumn{2}{|c|}{ PS-206k, $T=180^{\circ} \mathrm{C}$} & \multicolumn{2}{|c|}{ PS-133k, $T=160{ }^{\circ} \mathrm{C}$} & \multicolumn{2}{|c|}{ PS- $185 \mathrm{k}, T=160^{\circ} \mathrm{C}$} & \multicolumn{2}{|c|}{ PS-200k, $T=160^{\circ} \mathrm{C}$} \\
\hline & $g_{i}(\mathrm{~Pa})$ & $\tau_{i}(\mathrm{~s})$ & $g_{i}(\mathrm{~Pa})$ & $\tau_{i}(\mathrm{~s})$ & $g_{i}(\mathrm{~Pa})$ & $\tau_{i}(\mathrm{~s})$ & $g_{i}(\mathrm{~Pa})$ & $\tau_{i}(\mathrm{~s})$ \\
\hline 1 & $4.55 \mathrm{E}+0.6$ & $2.73 \mathrm{E}-06$ & $2.84 \mathrm{E}+07$ & $3.06 \mathrm{E}-06$ & $1.92 \mathrm{E}+07$ & $4.76 \mathrm{E}-06$ & $2.89 \mathrm{E}+05$ & $1.88 \mathrm{E}-03$ \\
\hline 2 & $4.40 \mathrm{E}+0.5$ & $3.27 \mathrm{E}-05$ & $6.68 \mathrm{E}+05$ & $7.70 \mathrm{E}-05$ & $5.49 \mathrm{E}+05$ & $1.02 \mathrm{E}-04$ & $5.26 \mathrm{E}+04$ & $1.90 \mathrm{E}-02$ \\
\hline 3 & $1.33 \mathrm{E}+05$ & $3.23 \mathrm{E}-04$ & $2.41 \mathrm{E}+05$ & $4.26 \mathrm{E}-04$ & $1.94 \mathrm{E}+05$ & $5.11 \mathrm{E}-04$ & $4.51 \mathrm{E}+04$ & $1.04 \mathrm{E}-01$ \\
\hline 4 & $6.10 \mathrm{E}+04$ & $4.19 \mathrm{E}-03$ & $1.04 \mathrm{E}+05$ & $1.95 \mathrm{E}-03$ & $8.99 \mathrm{E}+04$ & $2.10 \mathrm{E}-03$ & $5.15 \mathrm{E}+04$ & $4.83 \mathrm{E}-01$ \\
\hline 5 & $6.35 \mathrm{E}+04$ & $4.12 \mathrm{E}-02$ & $5.72 \mathrm{E}+04$ & $8.72 \mathrm{E}-03$ & $4.95 \mathrm{E}+04$ & $9.27 \mathrm{E}-03$ & $5.22 \mathrm{E}+04$ & $2.06 \mathrm{E}+00$ \\
\hline 6 & $7.97 \mathrm{E}+04$ & $3.45 \mathrm{E}-01$ & $4.76 \mathrm{E}+04$ & $3.66 \mathrm{E}-02$ & $4.08 \mathrm{E}+04$ & $4.13 \mathrm{E}-02$ & $4.06 \mathrm{E}+04$ & $5.43 \mathrm{E}+00$ \\
\hline 7 & $2.28 \mathrm{E}+04$ & 1.234 & $4.95 \mathrm{E}+04$ & $1.41 \mathrm{E}-01$ & $4.05 \mathrm{E}+04$ & $1.65 \mathrm{E}-01$ & - & - \\
\hline 8 & - & - & $3.78 \mathrm{E}+04$ & $4.60 \mathrm{E}-01$ & $4.52 \mathrm{E}+04$ & $5.93 \mathrm{E}-01$ & - & - \\
\hline 9 & - & - & $5.63 \mathrm{E}+04$ & $1.11 \mathrm{E}+00$ & $5.32 \mathrm{E}+04$ & $2.36 \mathrm{E}+00$ & - & - \\
\hline 10 & - & - & - & - & $1.93 \mathrm{E}+04$ & $4.60 \mathrm{E}+00$ & - & - \\
\hline
\end{tabular}

We identify here $\tau_{d}$ with the mean quadratic average of the relaxation times of the discrete relaxation spectrum and calculate $\eta_{0}$ from the relaxation spectrum:

$$
\tau_{d}=\frac{\sum_{i} g_{i} \tau_{i}^{2}}{\sum_{i} g_{i} \tau_{i}}
$$

Table 4 displays the plateau modulus, Rouse relaxation time, disengagement time, zero-shear viscosity, number of entanglements, and number of Kuhn segments between entanglements of the samples as described in Eqs. (2), (3) and (6).

\section{The extended interchain pressure model}

The tube model of Doi and Edwards (1986) assumes that the diameter of the tube $a_{0}$ is constant; i.e. the tension in the macromolecular chain remains constant and equal to its equilibrium value even for nonlinear deformations. The extra stress tensor $\sigma(t)$ is then the consequence of the orientation of tube segments due to the flow. The resulting constitutive equation is of the single integral form, if the tube segments are assumed to align independently of each other in the flow field (i.e. the "independent alignment (IA)" approximation):

$\boldsymbol{\sigma}(t)=\int_{-\infty}^{t} \frac{\partial G\left(t-t^{\prime}\right)}{\partial t^{\prime}} \mathbf{S}_{\mathrm{DE}}^{\mathrm{IA}}\left(t, t^{\prime}\right) d t^{\prime}$

$G(t)$ is the linear-viscoelastic shear relaxation modulus, and the relative strain measure $\mathbf{S}_{\mathrm{DE}}^{\mathrm{IA}}$ is given by

$\mathbf{S}_{\mathbf{D E}}^{\mathbf{I A}}\left(t, t^{\prime}\right) \equiv 5\left\langle\frac{\mathbf{u}^{\prime}\left(t, t^{\prime}\right) \mathbf{u}^{\prime}\left(t, t^{\prime}\right)}{u^{\prime 2}}\right\rangle=5 \mathbf{S}\left(t, t^{\prime}\right)$

$\mathbf{S}$ is the relative second-order orientation tensor. The bracket denotes an average over an isotropic distribution of end-to-

\begin{tabular}{|c|c|c|c|c|c|c|}
\hline \multirow[b]{2}{*}{ Parsimonious spectrum, $i$} & \multicolumn{2}{|c|}{ PS-285k/2k-65, $T=150{ }^{\circ} \mathrm{C}$} & \multicolumn{2}{|c|}{ PS-285k/2k-47, $T=140{ }^{\circ} \mathrm{C}$} & \multicolumn{2}{|c|}{ PS-545k//1k-52, $T=130^{\circ} \mathrm{C}$} \\
\hline & $g_{i}(\mathrm{~Pa})$ & $\tau_{i}(\mathrm{~s})$ & $g_{i}(\mathrm{~Pa})$ & $\tau_{i}(\mathrm{~s})$ & $g_{i}(\mathrm{~Pa})$ & $\tau_{i}(\mathrm{~s})$ \\
\hline 1 & $3.88 \mathrm{E}+06$ & $1.21 \mathrm{E}-05$ & $1.41 \mathrm{E}+07$ & $3.09 \mathrm{E}-06$ & $4.50 \mathrm{E}+06$ & $3.30 \mathrm{E}-06$ \\
\hline 2 & $1.58 \mathrm{E}+05$ & $2.22 \mathrm{E}-04$ & $2.04 \mathrm{E}+05$ & $9.21 \mathrm{E}-05$ & $1.47 \mathrm{E}+05$ & $1.06 \mathrm{E}-04$ \\
\hline 3 & $6.14 \mathrm{E}+04$ & $1.22 \mathrm{E}-03$ & $7.57 \mathrm{E}+04$ & $4.55 \mathrm{E}-04$ & $1.42 \mathrm{E}+04$ & $4.97 \mathrm{E}-01$ \\
\hline 4 & $2.58 \mathrm{E}+04$ & $6.51 \mathrm{E}-03$ & $3.61 \mathrm{E}+04$ & $1.99 \mathrm{E}-03$ & 4.17E+04 & $1.04 \mathrm{E}-03$ \\
\hline 5 & $1.76 \mathrm{E}+04$ & $3.14 \mathrm{E}-02$ & $1.87 \mathrm{E}+04$ & $1.01 \mathrm{E}-02$ & $1.47 \mathrm{E}+04$ & $9.68 \mathrm{E}-03$ \\
\hline 6 & $1.78 \mathrm{E}+04$ & $1.36 \mathrm{E}-01$ & $1.16 \mathrm{E}+04$ & $4.99 \mathrm{E}-02$ & $1.22 \mathrm{E}+04$ & $7.53 \mathrm{E}-02$ \\
\hline 7 & $2.14 \mathrm{E}+04$ & $5.93 \mathrm{E}-01$ & $1.06 \mathrm{E}+04$ & $2.05 \mathrm{E}-01$ & $1.52 \mathrm{E}+04$ & $3.01 \mathrm{E}+00$ \\
\hline 8 & $2.36 \mathrm{E}+04$ & $2.70 \mathrm{E}+00$ & $1.09 \mathrm{E}+04$ & $8.05 \mathrm{E}-01$ & $1.27 \mathrm{E}+04$ & $1.35 \mathrm{E}+01$ \\
\hline 9 & $4.34 \mathrm{E}+03$ & $6.07 \mathrm{E}+00$ & $1.21 \mathrm{E}+04$ & $2.86 \mathrm{E}+00$ & - & - \\
\hline 10 & - & - & $3.04 \mathrm{E}+02$ & $1.16 \mathrm{E}+01$ & - & - \\
\hline
\end{tabular}

Table 3 Parsimonious spectra of PS solution samples by IRIS software (Winter and Mours 2006) 
Table 4 Material properties of polystyrene melts and solutions in oligomeric styrene at temperatures given in Table 1

\begin{tabular}{lllllll}
\hline Sample name & $G_{N}[\mathrm{~Pa}]$ & $\tau_{d}[\mathrm{~s}]$ & $\tau_{R}[\mathrm{~s}]$ & $\eta_{0}[\mathrm{~Pa} \mathrm{~s}]$ & $Z$ & $N_{e}$ \\
\hline PS-206k & - & 0.76 & 0.056 & $5.86 \mathrm{E}+04$ & 15.5 & 21.8 \\
PS-133k & $2.79 \mathrm{E}+05$ & 0.87 & 0.16 & $8.92 \mathrm{E}+04$ & 10 & 21.8 \\
PS-185k & $2.58 \mathrm{E}+05$ & 2.88 & 0.29 & $2.50 \mathrm{E}+05$ & 13.9 & 21.8 \\
PS-200k & - & 3.98 & 0.37 & $3.59 \mathrm{E}+05$ & 15.2 & 21.8 \\
PS-285/2k-65 & $1.04 \mathrm{E}+05$ & 3.20 & 0.30 & $1.06 \mathrm{E}+05$ & 13.9 & 33.6 \\
PS-285/2k-47 & $5.80 \mathrm{E}+04$ & 2.94 & 0.44 & $5.00 \mathrm{E}+04$ & 10.1 & 46.3 \\
PS-545k/1k-52 & $6.76 \mathrm{E}+04$ & 10.87 & 0.56 & $2.25 \mathrm{E}+05$ & 21.3 & 41.9 \\
\hline
\end{tabular}

end unit vectors $\mathbf{u}\left(t^{\prime}\right)$ at creation time $t^{\prime}$ of the entanglements by reptation, and can be expressed as a surface integral over the unit sphere:

$$
\langle\rangle \equiv \frac{1}{4 \pi} \oiint[] \sin \theta d \theta d \varphi
$$

At the observation time $t$, the unit vectors $\mathbf{u}\left(t^{\prime}\right)$ are deformed to vectors $\mathbf{u}^{\prime}\left(t, t^{\prime}\right)$, which are calculated from the affine deformation hypothesis (with $\mathbf{F}^{-1}\left(t, t^{\prime}\right)$ being the relative deformation gradient tensor) as

$\mathbf{u}^{\prime}\left(t, t^{\prime}\right)=\mathbf{F}^{-1}\left(t, t^{\prime}\right) \cdot \mathbf{u}\left(t^{\prime}\right)$

$u^{\prime}=\left|\mathbf{u}^{\prime}\right|$ indicates the length of the vector $\mathbf{u}^{\prime}$.

The DE model does not account for any strain hardening. Doi and Edwards (1986) added a stretch process with a stretch $\lambda$ of the tube segments to their model in order to explain the discrepancies of the DE theory at start-up of extensional flows. Pre-averaging the stretch, i.e. assuming that the stretch is uniform along the chain contour length and an explicit function of the observation time $\lambda(t)$, the extra stress tensor is given by

$$
\boldsymbol{\sigma}(t)=\lambda^{2}(t) \int_{-\infty}^{t} \frac{\partial G\left(t-t^{\prime}\right)}{\partial t^{\prime}} \mathbf{S}_{\mathrm{DE}}^{\mathrm{IA}}\left(t, t^{\prime}\right) d t^{\prime}
$$

Equation (11) required finding a stretch evolution equation, and a vast variety of concepts based on different kinetic ideas have been proposed in recent years [see e.g. Doi and Edwards 1986, Doi and Edwards 1986, Pearson et al. 1989, McLeish and Larson 1998, Mead et al. 1995]. However, it should be noted that Eq. (11) with any function $\lambda^{2}(t)$ is not in agreement with experimental results of reversed elongational flow of a monodisperse polystyrene melt (Nielsen et al. 2008).

While in models with pre-averaged stretch, the tube diameter is always assumed to stay constant and equal to its equilibrium value $a_{0}$, stretch can also be introduced by the assumption of a strain-dependent tube diameter, as first suggested by Marrucci and de Cindio (1980). Accordingly, the pre-averaging of the stretch can be avoided as well, which is inherently present in models based on Eq. (11) or its differential approximations. A generalized tube model with strain-dependent tube diameter was presented by Wagner and Schaeffer (1992) (Wagner and Schaeffer 1992a; Wagner and Schaeffer 1993; Wagner and Schaeffer 1994, and Wagner et al. 2001; Wagner et al. 2001). In the molecular stress function (MSF) theory, tube segment stretch $f=f\left(t, t^{\prime}\right)$ is the inverse of the relative tube diameter $a$ :

$f\left(t, t^{\prime}\right)=a_{0} / a\left(t, t^{\prime}\right)$

which decreases from its equilibrium value $a_{0}$ with increasing stretch. Considering that the tube diameter $a$ represents the mean field of the surrounding chains, it is assumed that the tube diameter is independent of tube segment orientation. The extra stress is then given as

$\boldsymbol{\sigma}(t)=\int_{-\infty}^{t} \frac{\partial G\left(t-t^{\prime}\right)}{\partial t^{\prime}} f^{2} \mathbf{S}_{D E}^{I A}\left(t, t^{\prime}\right) d t^{\prime}$

In contrast to Eq. (11), stretch in Eq. (13) does not only depend on the observation time $t$, but depends on the strain history: for time-dependent strain histories, chain segments with long relaxation times (i.e. at the centre of the chain) experience higher stretches than chain segments with short relaxation times (i.e. at the chain ends).

Based on the "interchain tube pressure" concept of Marrucci and Ianniruberto (2004), Wagner and RolónGarrido (2009b) and Wagner and Rolón-Garrido (2009a) developed an extended interchain pressure (EIP) model for monodisperse polystyrene melts consisting of Eq. (13) and an evolution equation for the molecular stretch $f$ :

$\frac{\partial f}{\partial t}=f(\kappa: \mathbf{S})-\frac{1}{3} \frac{f-1}{\tau_{R}}-\frac{2}{3} \frac{f^{2}\left(f^{3}-1\right)}{3 \tau_{R}}$

with the initial conditions $f\left(t=t^{\prime}, t^{\prime}\right)=1$. The first term on the right-hand side represents an on average affine stretch rate with $\kappa$ the velocity gradient tensor, the second term takes into account Rouse relaxation with Rouse time $\tau_{R}$ in the longitudinal direction of the tube and the third term limits molecular stretch due to the interchain tube pressure in the lateral direction of a tube segment with tube diameter relaxation time $\tau_{a}=$ $3 \tau_{R}$. It should be noted that in the limit of small stretch, i.e. for $f-1<<1$, the interchain pressure term reduces to $f^{2}\left(f^{3}-1\right)$ $\cong 3(f-1)$ and the classical relation of Pearson et al. (1989) is recovered from Eq. (14): 
$\frac{\partial f}{\partial t}=f(\kappa: \mathbf{S})-\frac{1}{\tau_{R}}(f-1)$

Predictions of Eqs. (13) and (14) were shown to be in excellent agreement with available elongational flow data of PS melts and solutions (Narimissa et al. 2020). In the following, we test the applicability of the EIP model to shear flow.

\section{Comparison of EIP model predictions with shear flow data of PS-206k melt}

Figure 1 shows the comparison between the transient shear data of melt PS-206k at $180^{\circ} \mathrm{C}$ (Schweizer et al. 2008) and the EIP model. Step shear rate tests were performed in the range $0.05 s^{-1} \leq \gamma \leq 47.16 s^{-1}$ at $180{ }^{\circ} \mathrm{C}$, and in the range of $0.2 s^{-1} \leq$ $\dot{\gamma} \leq 47.16 \mathrm{~s}^{-1}$ at $160{ }^{\circ} \mathrm{C}$. The data measured at $160{ }^{\circ} \mathrm{C}$ were time-temperature shifted to $180{ }^{\circ} \mathrm{C}$, corresponding to shear rates of $1.92 s^{-1} \leq \dot{\gamma} \leq 452 s^{-1}$. We restrict attention here to the stress growth coefficients of the shear stress, $\eta^{+}(t)$, and the first normal stress difference, $\psi_{1}^{+}(t)$. Measurements of the second normal stress difference with CPP tools scatter severely mirroring the instabilities of the free surface at the rim as discussed in (Schweizer et al. 2008) and will not be considered here. The EIP model predictions were calculated by Eqs. (13) and (14) using the parsimonious relaxation spectrum (Table 3 ) and a Rouse time $\tau_{R}=0.056 \mathrm{~s}$ (Table 4 ) at the test temperature $\left(T=180^{\circ} \mathrm{C}\right)$. Model predictions are in quantitative agreement with the overshoot of the shear stress growth coefficient $\eta^{+}(t)$ for shear rates up to $48 \mathrm{~s}^{-1}$, while at shear rates of $192 \mathrm{~s}^{-1}$ and $452 \mathrm{~s}^{-1}$, the experimental data show a time delay relative to the predictions (Fig. 1). This is due to a time delay in the effective motor motion of the rheometer as discussed in (Schweizer et al. 2008). While the agreement between the viscosity overshoot of data and model can be rated as excellent in the investigated shear rate range, the steady-state shear viscosity data are increasingly overpredicted for shear rates $\dot{\gamma} \geq 10 s^{-1}$. The experimental time delay relative to the model predictions in the rise time of the first normal stress coefficient $\psi_{1}^{+}(t)$ is mostly instigated by the limited stiffness of the shear rheometer in the axial direction causing radial inflow of the melt (Meissner 1972). The agreement between steady-state data of $\psi_{1}$ and model is quantitative only for $\dot{\gamma}<10 s^{-1}$, while the model again increasingly overpredicts the steady-state values of $\psi_{1}$ with increasing shear rate. At higher shear rates, a shallow overshoot of $\psi_{1}^{+}(t)$ is predicted which is in qualitative agreement with the data.

In order to improve the shear flow predictions of the EIP model, we will incorporate the constraint release $C R_{2}$ relaxation mechanism into the EIP model and compare its predictions with the PS-206k data in the following section.

\section{Introducing $C R_{2}$ in the EIP model}

The constraint release relaxation mechanism $C R_{2}$ is based on the convective constrain release relaxation (CCR) mechanism in polydisperse polymers which was first introduced by Marrucci (Marrucci 1996) and Ianniruberto and Marrucci (Ianniruberto and Marrucci 1996). In brief, the CCR mechanism explains the disentanglement (i.e. the removal of the topological constraints) as a result of flow in the nonlinear regime of shear deformation $(W i>1)$. However, the CCR in its original form results in "hypothetical" creation of constraints/ entanglements (i.e. negative CCR) during flow at high strains as well as in the case of reverse flow (for more details, see Narimissa and Wagner 2016, Narimissa and Wagner 2019). Wagner et al. (2001) and later Narimissa and Wagner (2016) implemented $C R_{2}$ in the evolution equation of stretch using a specific combination of the second-order Rivlin-Ericksen tensors in such way that $\mathrm{CR}_{2}$ is only active in shear flows, and the theoretical creation of constraints is avoided in $C R_{2}$. Therefore, we introduce a $C R_{2}$ to the Rouse relaxation time of the evolution equation of stretch for melts (Eq. (14)),

$\frac{\partial f}{\partial t}=f(\kappa: \mathbf{S})-\frac{1}{3} \frac{f-1}{\tau_{R C R}}-\frac{2}{3} \frac{f^{2}\left(f^{3}-1\right)}{3 \tau_{R C R}}$

Here, $\tau_{R C R}$ is defined as:

$\frac{1}{\tau_{R C R}}=\frac{1}{\tau_{R}}+\beta C R_{2}$

$C R_{2}$ is the result of different convection mechanisms for tube orientation and tube cross section, and constant strainrate flow (for more detail, see review Narimissa and Wagner 2019) is given by:

$C R_{2}=\frac{1}{2} \sqrt{\left|\mathbf{A}_{2}: \mathbf{S}-\mathbf{A}_{1}^{2}: \mathbf{S}\right|}=\sqrt{|\mathbf{W} \cdot \mathbf{D}: \mathbf{S}|}$

Here, $\mathbf{A}_{1}^{2}=4 \mathbf{D}^{\mathbf{2}}$ and $\mathbf{A}_{2}=\frac{\partial \mathbf{A}_{1}}{\partial t}+\mathbf{A}_{\mathbf{1}}^{\mathbf{2}}+2\left(\mathbf{W} \cdot \mathbf{D}+\mathbf{D} \cdot \mathbf{W}^{\mathbf{T}}\right)$ are the second-order Rivlin-Ericksen tensors, and $\mathbf{D}=\frac{1}{2}$ $\left(\kappa+\kappa^{\mathbf{T}}\right)$ and $\mathbf{W}=\frac{1}{2}\left(\kappa-\kappa^{\mathbf{T}}\right)$ are the rate of deformation and rotation tensor, respectively. As shown in the Appendix, $C R_{2}$ can also be expressed in terms of the persistence-of-straining tensor as proposed by Thompson and Souza Mendez (Thompson and Souza Mendes 2005a; Thompson and Souza Mendes 2005b). In simple shear flow, $C R_{2}$ becomes,

$C R_{2}=\frac{1}{2} \sqrt{\dot{\gamma}^{2}\left|S_{11}-S_{22}\right|}$

$\beta$ (in Eq. (17)) is a free parameter required to obtain agreement with the stress growth coefficient and first normal stress function measurements. 
Figure 2 shows the comparison between the predictions of the EIP model with $C R_{2}$ (Eqs. (13), (16), (17) and (19)) and the data of the growth coefficients of shear stress and normal stress of melt PS-206k with $\beta=2$. The incorporation of $C R_{2}$ in EIP model improves the predictions of the steady-state shear viscosity and the first normal stress coefficient, and agreement between the model and data is nearly quantitative up to $\dot{\gamma}=50 \mathrm{~s}^{-1}$. However, the underprediction of the viscosity overshoots at early $\eta^{+}(t)$ shows progressive deterioration at
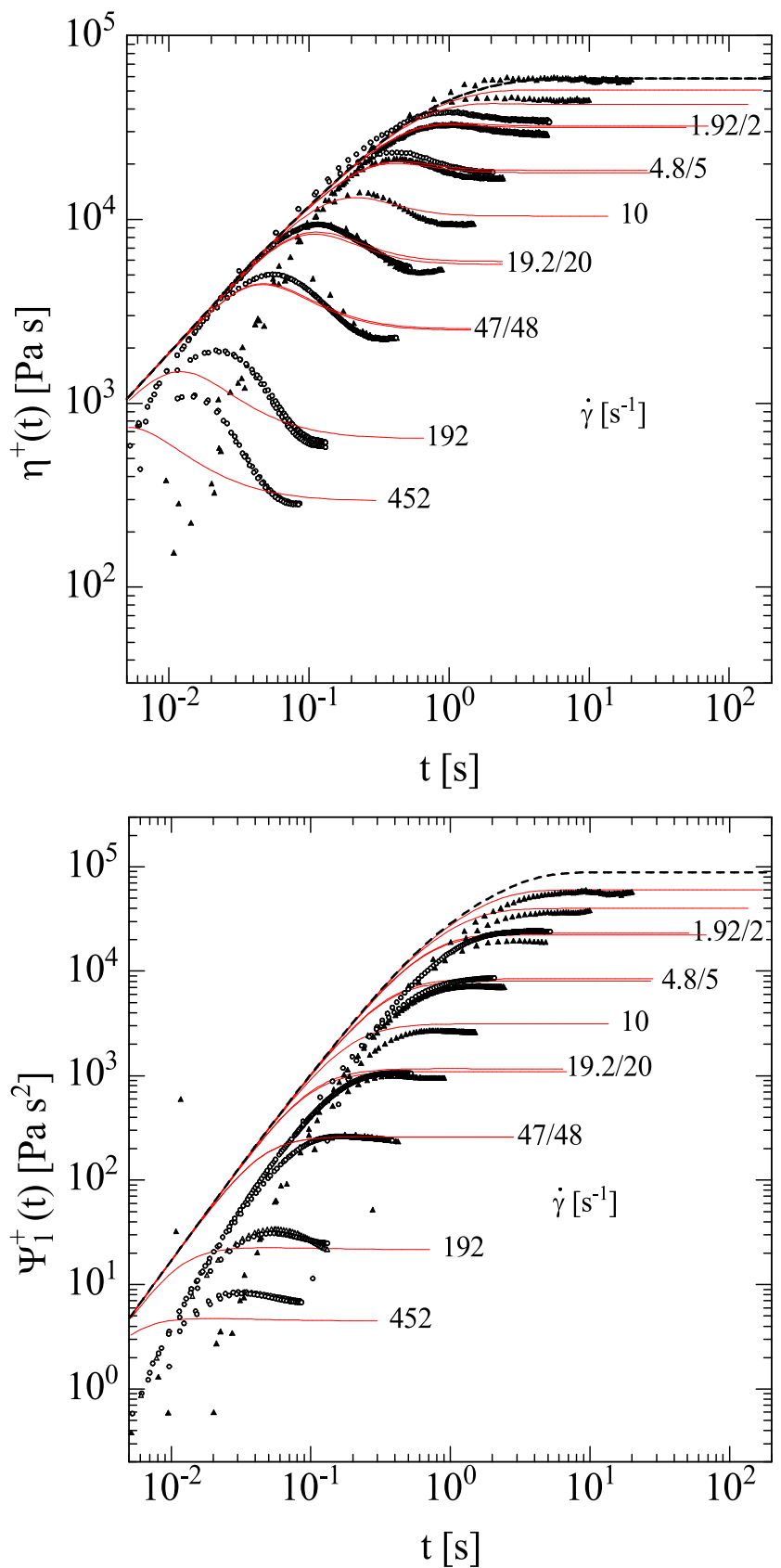

Fig. 2 Comparison between the predictions (red lines) of the EIP model with $C R_{2}$ (Eqs. (13), (16), (17) and (19)) with $\beta=2$ and the shear stress growth coefficient $\eta^{+}(t)$ and the first normal stress growth coefficient $\psi_{1}^{+}$ $(t)$ of PS-206k at $180^{\circ} \mathrm{C}$ increasing shear rates. The underpredictions are even more evident in the case of the first normal stress growth coefficient at $\dot{\gamma}>50 s^{-1}$ where the model is incapable of predicting any significant overshoot. In brief, at shear rates $\dot{\gamma}>50 \mathrm{~s}^{-1}$, the constraint release parameter suppresses the overshoot predictions in both $\eta^{+}(t)$ and $\psi_{1}^{+}(t)$; yet, it results in improved predictions of the steady-state data.

At increasing values of $\beta$, the stretch evolution in Eq. (16) is effectively suppressed, i.e. $f_{i}\left(t, t^{\prime}\right) \cong 1$, as enhanced Rouse relaxation and interchain pressure prevent any stretch. The EIP- $C R_{2}$ model reduces to the original Doi-Edwards model (Doi and Edwards 1978a; Doi and Edwards 1978b; Doi and Edwards 1979; Doi and Edwards 1986) with independent alignment (DEIA). Figure 3 illustrates the predictions of the DEIA model, Eq. (7), of the melt data of PS-206k. The DEIA model agrees quantitatively with the experimental data of the steady-state shear viscosity and the first normal stress coefficient while the overshoot in the shear stress growth coefficient $\eta^{+}(t)$ is increasingly underpredicted with increasing shear rates, and no overshoot is predicted in the first normal stress growth coefficient $\psi_{1}^{+}(t)$. This is in line with earlier findings (Narimissa and Wagner 2018) that the DEIA model can produce quantitative predictions for the weak maxima (only in $\eta^{+}(t)$ ) and steady states (in both $\eta^{+}(t)$ and $\psi_{1}^{+}(t)$ ) seen for linear polydisperse melts at shear rates $\dot{\gamma} \leq 10 \mathrm{~s}^{-1}$.

We remind that shear stress overshoot in the DEIA model is an orientational effect, which is caused by the 12component of the orientation tensor (Doi and Edwards 1979),

$S_{12}=\left\langle\frac{u_{1}^{\prime} u_{2}^{\prime}}{u^{\prime 2}}\right\rangle$

$S_{12}$ first increases with shear deformation, then goes through a maximum at $\gamma=2.1$ (see e.g. (Wagner and RolónGarrido 2010) and finally approaches zero when the tube segment is fully aligned in the shear plane. No stress overshoot in $\psi_{1}^{+}(t)$ is predicted by the DEIA model because the difference between the 11 and the 22 components of the orientation tensor increases monotonously to a value of $S_{11}-S_{22}=1$ (full alignment in the shear plane) with increasing shear deformation $\gamma$ (Doi and Edwards 1979),

$S_{11}-S_{22}=\gamma S_{12}$

Therefore, we can conclude that while the DEIA model allows for quantitative description of the steady-state values of shear viscosity and first normal stress coefficient, the EIP model (Eqs. (13)) with stretch evolution Eq. (14) provides excellent prediction of the stress overshoot of both the stress growth coefficients of the shear stress, $\eta^{+}(t)$ and the first normal stress difference, $\psi_{1}^{+}(t)$. It is important to note that in contrast to extensional flows, stretch evolution in shear flows goes through a maximum 

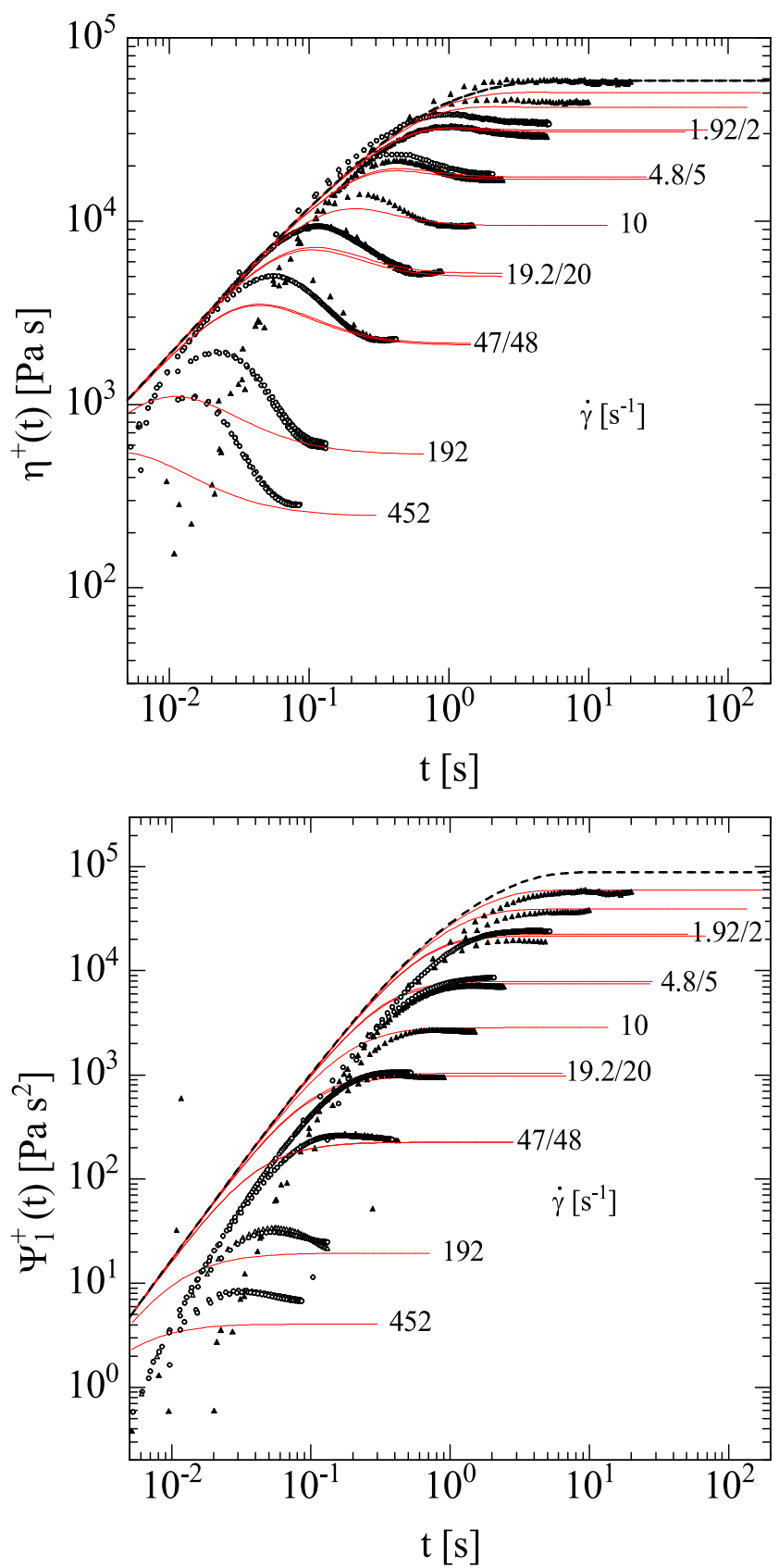

Fig. 3 Comparison between the predictions (red lines) of the DE model (Eq. (13) with $f_{i}\left(t, t^{\prime}\right) \cong 1$ ) and the shear stress growth coefficient $\eta^{+}(t)$ and the first normal stress growth coefficient $\psi_{1}^{+}(t)$ of PS-206k at $180{ }^{\circ} \mathrm{C}$

(see e.g. Wagner and Rolón-Garrido 2010): The stretch evolution Eq. (14) in shear flow is given by

$\frac{\partial f}{\partial t}=\dot{\gamma} S_{12}-\frac{1}{3} \frac{f-1}{\tau_{R}}-\frac{2}{3} \frac{f^{2}\left(f^{3}-1\right)}{3 \tau_{R}}$

and since $S_{12}$ goes through a maximum, the stretch $f$ also goes through a maximum, although this maximum is shifted to larger shear deformations with increasing shear rate. While the shear stress overshoot is the result of orientational stress overshoot and stretch overshoot combined, the normal stress overshoot depends solely on the overshoot in stretch. Based on these considerations, we propose in the following a novel constitutive model consisting of a combination of the DEIA and the EIP model.

\section{New constitutive approach for rotational flows}

We begin by considering that for flows starting at $t=0$, the extra stress tensor is given by

$$
\begin{aligned}
\boldsymbol{\sigma}(t)= & G(t) f^{2}(t, 0) \mathbf{S}_{D E}^{I A}(t, 0) \\
& +\int_{0}^{t} \frac{\partial G\left(t-t^{\prime}\right)}{\partial t^{\prime}} f^{2}\left(t, t^{\prime}\right) \mathbf{S}_{D E}^{I A}\left(t, t^{\prime}\right) d t^{\prime}
\end{aligned}
$$

The first term on the right-hand side accounts for the contribution to the stress tensor of entanglements, which due to reptation, were created before the inception of flow, i.e. during the times $-\infty<t \leq 0$, and still exist at the time of observation at time $t$. These entanglements, which are located at the centre of the chain, experience the full stretch $f(t, 0)$ and the full orientation $\mathbf{S}_{D E}^{I A}(t, 0)$ between time $t=0$, when shear flow started, and the observation time $t$. The second (integral) term provides the stress contribution of entanglements, which were created by reptation at times $t^{\prime}$ during flow, i.e. in the time frame of $0<t^{\prime} \leq t$, and located more at the chain ends. Those entanglements experience the relative stretch $f\left(t, t^{\prime}\right)$ and the relative orientation $\mathbf{S}_{D E}^{I A}\left(t, t^{\prime}\right)$ between times $t^{\prime}$ and $t$. Based on the experimental observation of shear flow in the "Comparison of EIP model predictions with shear flow data of PS-206k melt" section, we assume now that rotational flow suppresses the stretch of entanglements created during flow, i.e. $f\left(t, t^{\prime}\right)=1$ for $0<t^{\prime} \leq t$, and therefore the stress is given by

$\boldsymbol{\sigma}(t)=G(t) f^{2}(t, 0) \mathbf{S}_{D E}^{I A}(t, 0)+\int_{0}^{t} \frac{\partial G\left(t-t^{\prime}\right)}{\partial t^{\prime}} \mathbf{S}_{D E}^{I A}\left(t, t^{\prime}\right) d t^{\prime}$

We argue that due to the rotational component of shear, entanglements created during flow at the chain ends do not see a reduction of the tube diameter; thus, they do not experience stretch. This is in line with the concept of "persistence-of-straining" developed by Thompson and Souza Mendez (Thompson and Souza Mendes 2005a, Thompson and Souza Mendes 2005b) as explained in the Appendix. While extensional flows feature a maximal intensity of persistence of straining, shear flow is a marginally weak flow. In contrast to entanglements created during shear flow, entanglements which already exist before inception of flow are stretched according to the evolution equation for $f(t, 0)$ : 
$\frac{\partial f(t, 0)}{\partial t}=f(t, 0)(\kappa: \mathbf{S}(t, 0))-\frac{1}{3} \frac{f(t, 0)-1}{\tau_{R}}-\frac{2}{3} \frac{f(t, 0)^{2}\left[f(t, 0)^{3}-1\right]}{3 \tau_{R}}$

with the initial condition $f(t=0,0)=1$. Equation (25) is obtained from Eq. (14) for the case of $t^{\prime}=0$. We note that for steady-state shear flow, the stress converges to the steady-state value of the Doi-Edwards IA Eq. (7):

$\boldsymbol{\sigma}(\infty)=\int_{-\infty}^{+\infty} \frac{\partial G\left(t-t^{\prime}\right)}{\partial t^{\prime}} \mathbf{S}_{D E}^{I A}\left(t, t^{\prime}\right) d t^{\prime}$

Therefore, Eqs. (24) and (25) combine a description of the stress overshoot of the shear viscosity (resulting from a combination of stretch and orientation) as well as the stress overshoot of the first normal stress coefficient (which is an effect of the stretch alone), with the steadystate viscosity of the DEIA model. The model does not require any nonlinear-viscoelastic parameter but relies solely on the linear-viscoelastic relaxation modulus $G(t)$ and the Rouse stretch relaxation time $\tau_{R}$ as defined in Eq. (27), which in turn is determined by linear viscoelasticity and the molar mass of the polystyrene melt.

A frame-indifferent formulation of Eqs. (24) and (25) can be obtained by considering the "flow strength" or "rotationality" of deformation (see Appendix).

\section{Comparison of new constitutive approach to experimental PS melt data}

In the following, we compare experimental data of Schweizer et al. (2008)) and Costanzo et al. (Costanzo et al. 2016; Costanzo et al. 2019) to predictions of Eqs. (24) and (25). The comparison between the predictions (lines) of our proposed model for shear flow and the PS melt data (symbols) are displayed in Figs. 4, 5, 6, 7, 8, 9 and 10.

\section{PS-206k (Schweizer et al. 2008)}

Excellent agreements are achieved between the predictions of Eqs. (24) and (25) and the stress growth coefficient $\eta^{+}(t)$ of PS-206k melt at $0.5 s^{-1} \leq \dot{\gamma} \leq 452 s^{-1}$ (Fig. 4). The observed signal-delay in the experimental data of $\eta^{+}(t)$ at higher shear rates $\dot{\gamma} \geq 192 s^{-1}$ is due to the finite acceleration of the rheometer (for more details, refer to Eq. 1 and Table 1 of (Schweizer et al. 2008)). Similarly, the observed signaldelay in the first normal stress coefficient $\psi_{1}^{+}(t)$ is due to the limited stiffness/compliance of the rheometer (Meissner 1972); when under the increasing normal force, the gap of the compliant rheometer opens and causes the radial inflow of the melt (Schweizer et al. 2008). Irrespective of the mentioned signal delays, the model achieves good agreement with
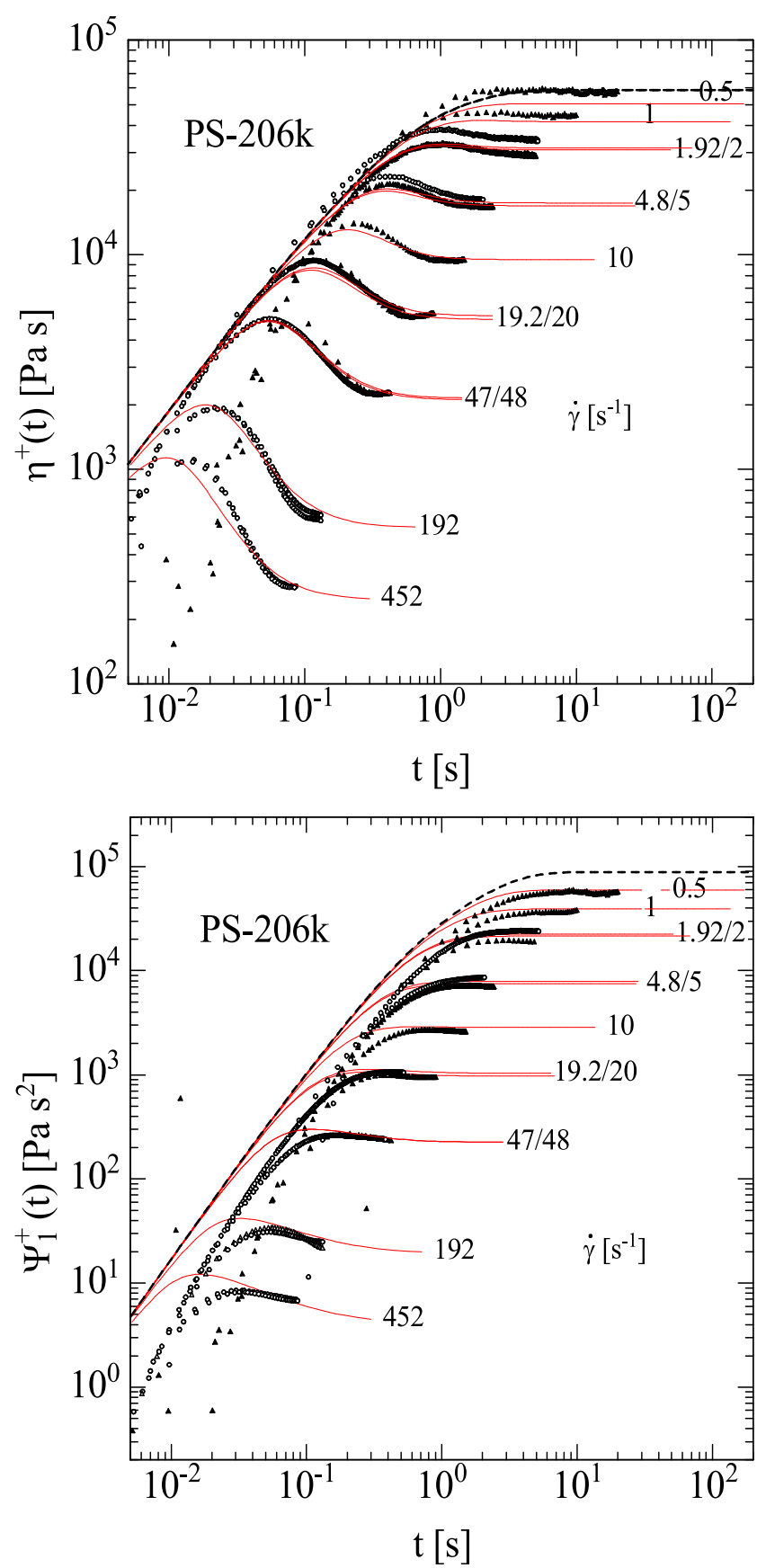

Fig. 4 Comparison between the predictions (red lines) of Eqs. (24) and (25) and the shear stress growth coefficient $\eta^{+}(t)$, and the first normal stress growth coefficient $\psi_{1}^{+}(t)$ of PS-206k at $180{ }^{\circ} \mathrm{C}$

the experimental data of the overshoot and steady-state values of $\psi_{1}^{+}(t)$ for PS-206k at higher shear rates (Fig. 4).

In the case of the steady-state shear flow, Fig. 5 shows quantitative agreements between the steady shear viscosity as well as first normal stress data and the predictions of Eqs. (24) and (25). This is in line with the Doi-Edwards (DEIA) model (Doi and Edwards 1978a, Doi and Edwards 1978b, Doi and Edwards 1979, Doi and Edwards 1986) providing quantitative prediction of steady shear flow. As explained in the 

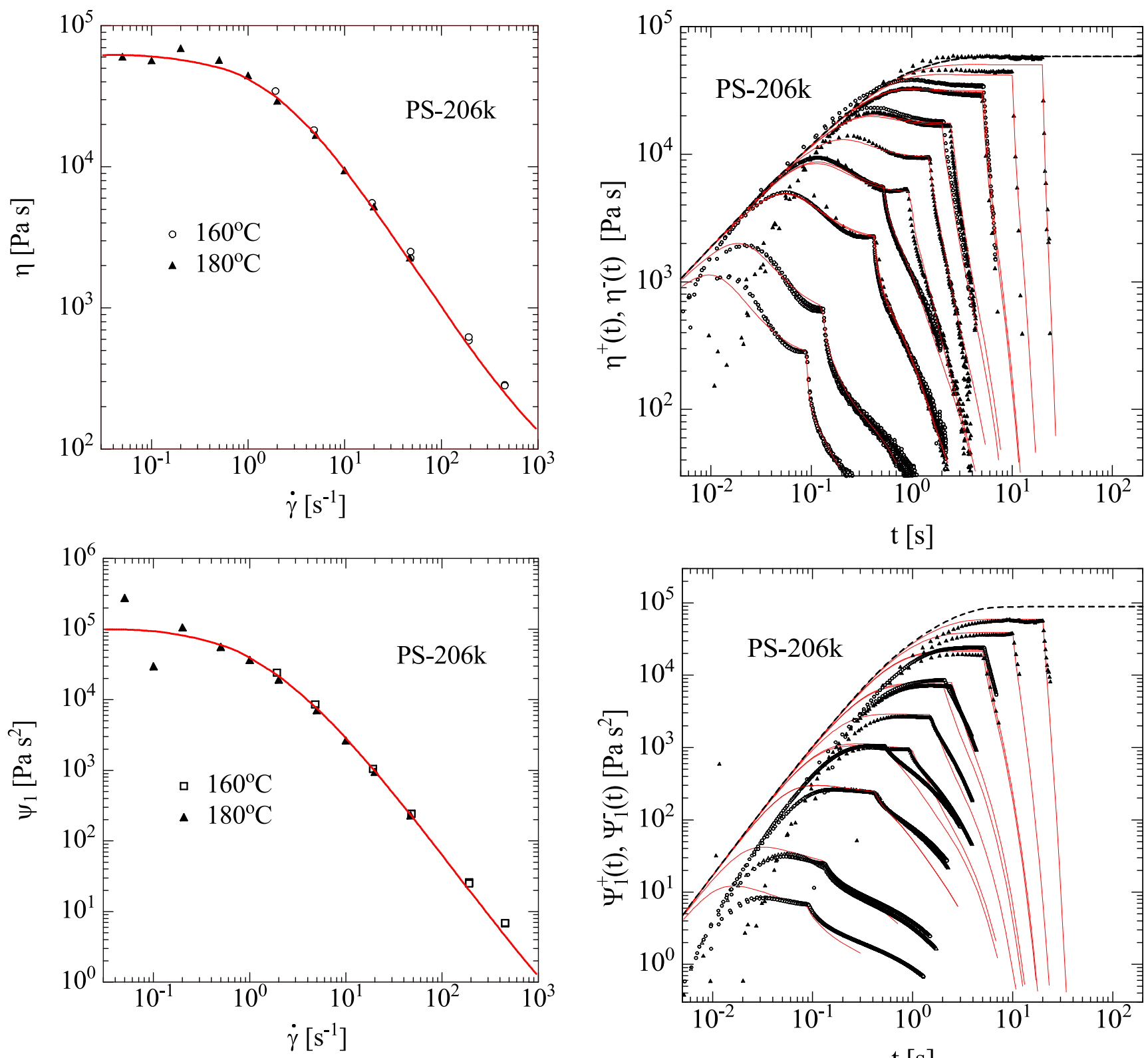

Fig. 5 Comparison between the predictions (red lines) of Eqs. (24) and (25) the steady-state shear viscosity $\eta$ and steady-state first normal stress coefficient $\psi_{1}$ of PS-206k at $180{ }^{\circ} \mathrm{C}$

Introduction, data of the second normal stress coefficient, as reported by Schweizer et al. (2008), show large uncertainty and will not be considered here.

Figure 6 shows the modelling of the stress relaxation after start-up of steady shear flow of PS206k by Eqs. (24) and (25). Quantitative agreement is achieved between model and experimental data of the shear stress relaxation coefficient $\eta^{-}(t)$ at all shear rates. Similar to the results seen in Fig. 4, the underprediction of the first normal stress relaxation coefficient $\psi_{1}^{-}(t)$ is due to the limited stiffness/compliance of the rheometer (Meissner 1972), with the difference that under a decreasing normal force, the gap of the compliant rheometer closes causing radial

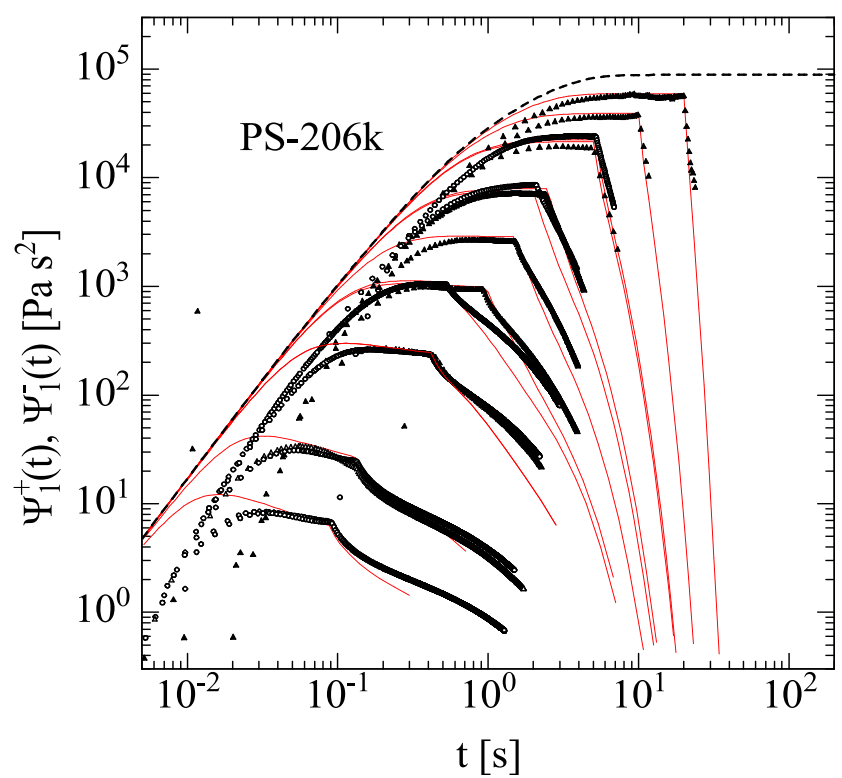

Fig. 6 Comparison between the predictions (red lines) of Eqs. (24) and (25) and the shear stress growth coefficient $\eta^{+}(t)$ and shear stress relaxation coefficient $\eta^{-}(t)$ as well as the first normal stress growth coefficient $\psi_{1}^{+}(t)$ and the first normal stress relaxation coefficient $\psi_{1}^{-}(t)$ of PS206k at $180^{\circ} \mathrm{C}$. Shear rates are identical to Fig. 4

outflow of the melt thereby retarding relaxation of the normal stress.

\section{PS-133k/ PS-185k/PS-200k melts (Costanzo et al. 2016, Costanzo et al. 2019)}

Figures 7, 8, 9 and 10 show the modelling of melt data of PS133k, PS-185k and PS-200k (Costanzo et al. 2016, Costanzo et al. 2019) obtained by different experimental set-ups (ARES rheometer, different CPP tools). The quantitative agreements 


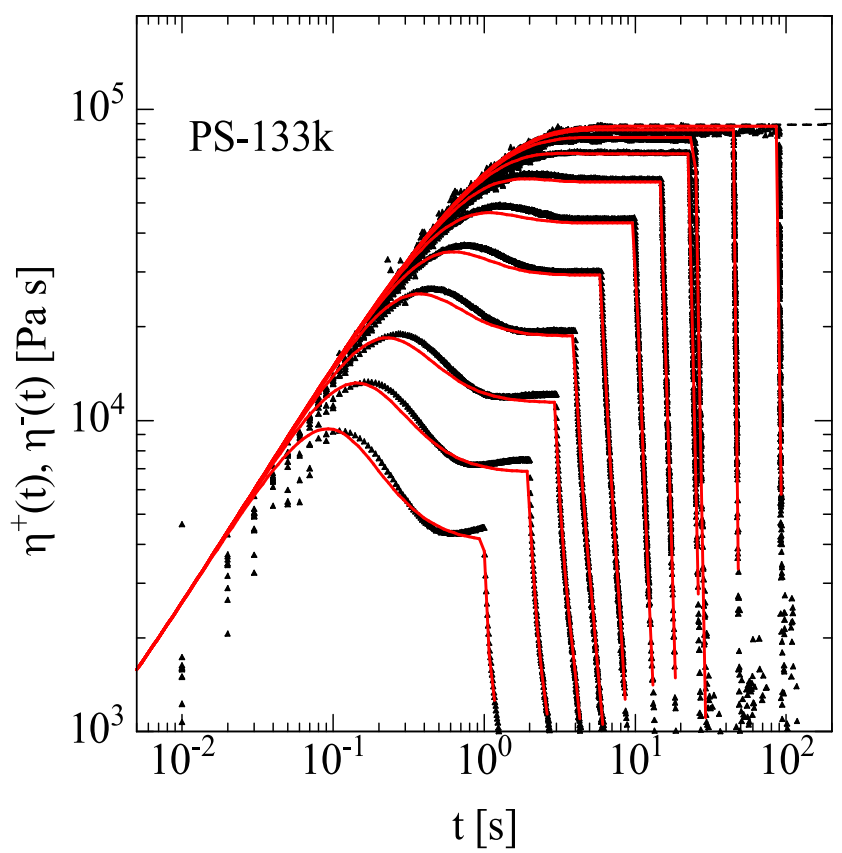

Fig. 7 Comparison between the predictions (red lines) of Eqs. (24) and (25) and the shear stress growth coefficient $\eta^{+}(t)$ and shear stress relaxation coefficient $\eta^{-}(t)$ of PS-133k at $160{ }^{\circ} \mathrm{C}$. Shear rates from top to bottom: $0.1,0.178,0.316,0.562,1,1.78,3.16,5.62,10,17.8$ and $31.6 \mathrm{~s}^{-1}$

achieved between the shear stress growth/relaxation coefficients of PS-133k (Fig. 7), PS-185k (Fig. 8) and PS-200k (Fig. 9) confirm the success of the model. The overpredictions

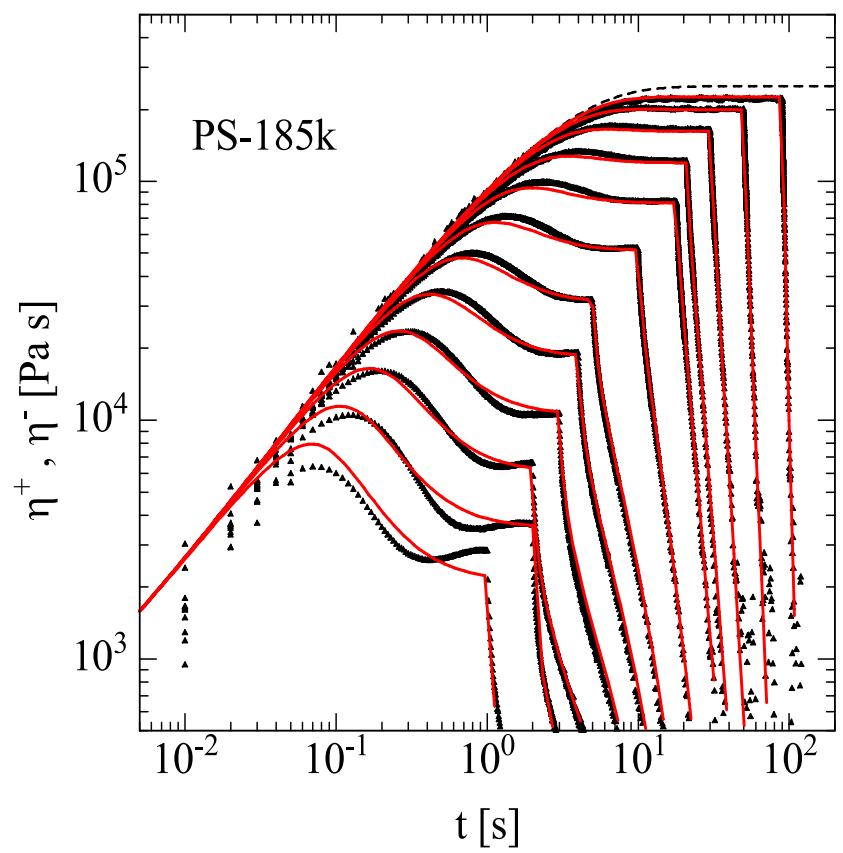

Fig. 8 Comparison between the predictions (red lines) of Eqs. (24) and (25) and the shear stress growth coefficient $\eta^{+}(t)$ and shear stress relaxation coefficient $\eta^{-}(t)$ of PS-185k at $160{ }^{\circ} \mathrm{C}$. Shear rates from top to bottom: $0.1,0.178,0.316,0.562,1,1.78,3.16,5.62,10,17.8,31.6$ and $56.2 \mathrm{~s}^{-1}$
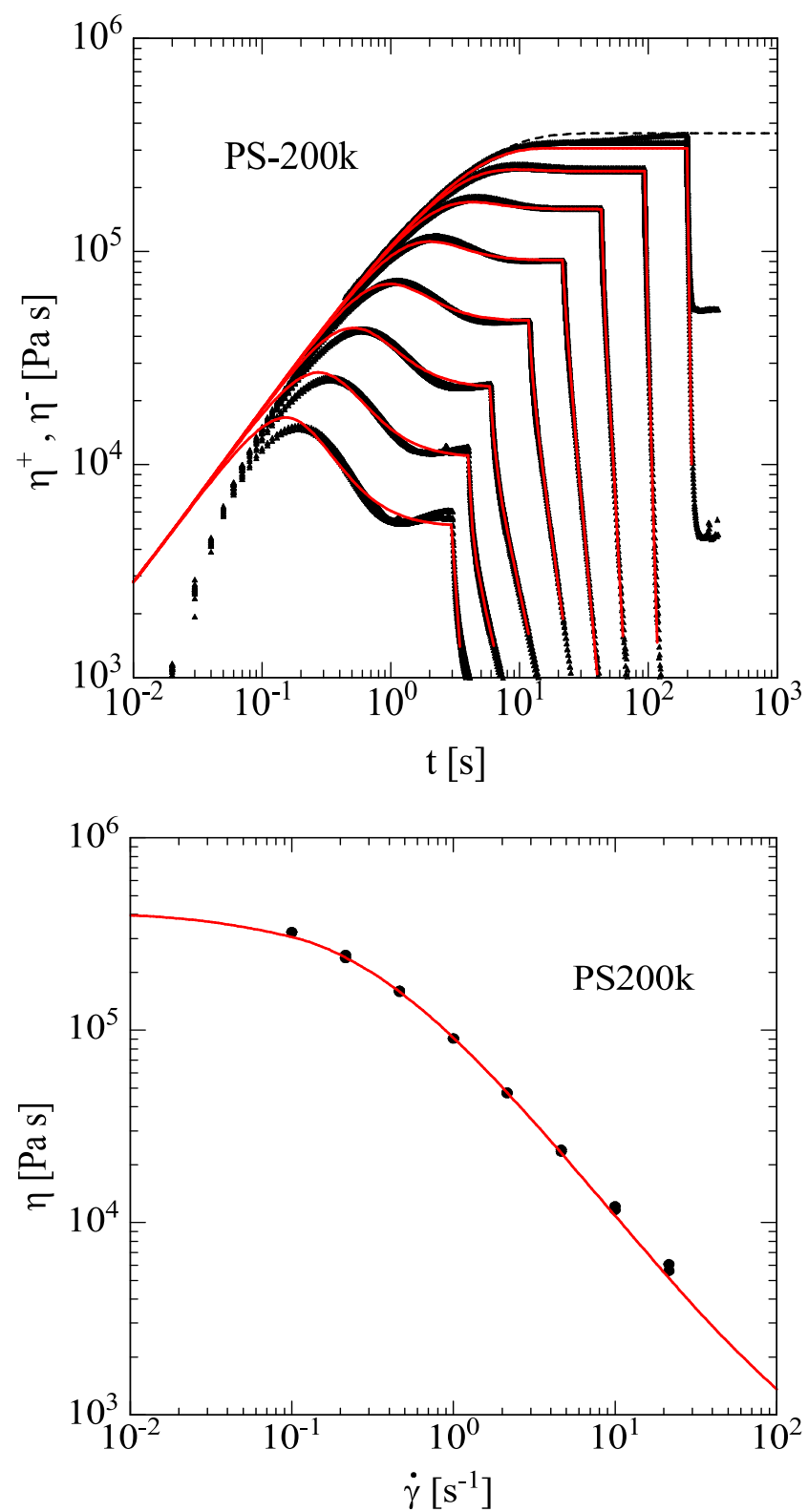

Fig. 9 Comparison between the predictions (red lines) of Eqs. (24) and (25) and the shear stress growth coefficient $\eta^{+}(t)$ and shear stress relaxation coefficient $\eta^{-}(t)$ as well as the steady-state shear viscosity $\eta$ of PS-200k at $160{ }^{\circ} \mathrm{C}$. Shear rates from top to bottom: 0.1, 0.215, 0.464, $1,2.15,4.64,10$ and $21.5 \mathrm{~s}^{-1}$

seen at higher shear rates in Fig. 8 is caused by the delay of the torque signal due to limited acceleration of rheometer drive (Schweizer et al. 2008). At shear strains larger than approximately 20, an increasing shear stress with increasing deformation is observed, creating undershoot of the shear stress. As already explained in the Introduction, we do not intend to model undershoot in this work.

Figure 10 shows the predictions of the model and the apparent normal stress growth/relaxation coefficients of PS$200 \mathrm{k}$. The apparent normal stress coefficient $\psi_{a}$ is obtained from the apparent normal stress $N_{a}$ : 


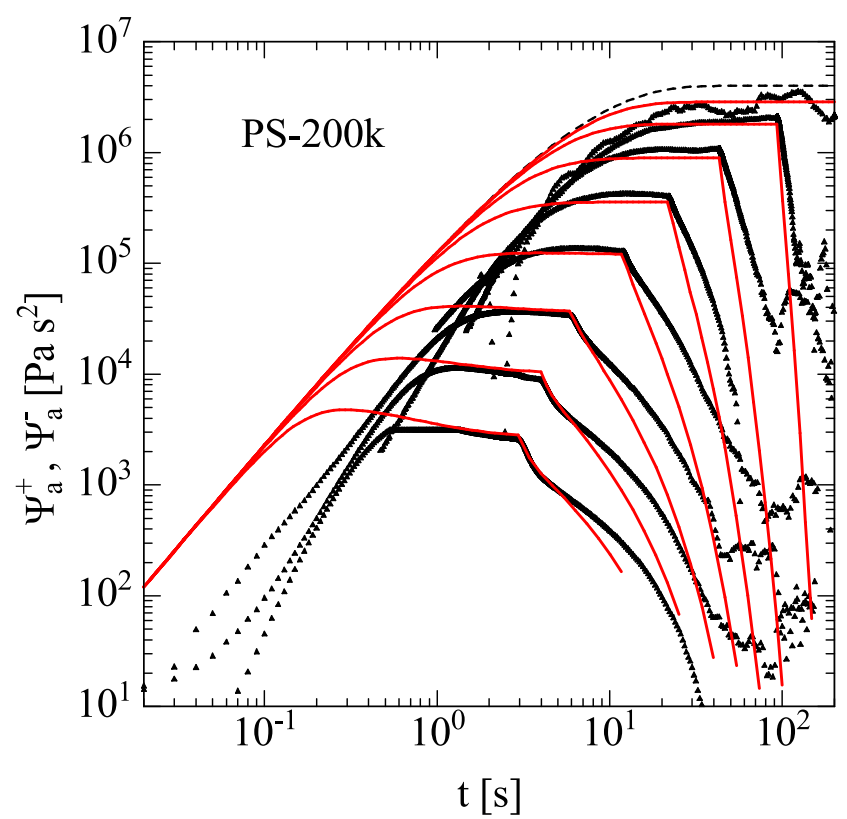

Fig. 10 Comparison between the predictions (red lines) of Eqs. (24) and (25) and the apparent normal stress growth coefficient $\psi_{a}^{+}(t)$ and the apparent normal stress relaxation coefficient $\psi_{a}^{-}(t)$ of PS-200k at $160{ }^{\circ} \mathrm{C}$. Shear rates from top to bottom: $0.1,0.215,0.464,1,2.15,4.64$, 10 and $21.5 \mathrm{~s}^{-1}$

$\psi_{\mathrm{a}}=\mathrm{N}_{\mathrm{a}} / \dot{\gamma}^{\cdot 2}$

Here, $N_{a}$ is defined in terms of the measured normal force $F$ of the inner partition with radius $R_{\text {stem }}$ and can be expressed in terms of the first normal stress difference $N_{1}$, the second normal stress difference $N_{2}$, and the sample radius $R$ (for more details, see Costanzo et al. 2018),

$\mathrm{N}_{\mathrm{a}}=\frac{2 F}{\pi R_{\text {stem }}^{2}}=N_{1}+2\left(N_{1}+2 N_{2}\right) \ln \left(\frac{R}{R_{\text {stem }}}\right)$

As $\left|N_{2}\right|<<N_{1}, N_{a}$ is mainly determined by $N_{1}$. For the start-up shear experiments shown in Fig. $10, R_{\text {stem }}=3 \mathrm{~mm}$ and $R=5.04 \mathrm{~mm}$. Considering the limited stiffness/ compliance of the rheometer causing a significant delay in the rise time of the normal force, good agreement between model predictions and the apparent normal stress coefficient data is achieved.

\section{Extension of the new constitutive approach to PS solutions}

Narimissa et al. (2020) showed that for PS solutions in oligomeric styrene, the stretch evolution equation (Eq. (14)) is obtained as: $\frac{\partial f}{\partial t}=f(\kappa: \mathbf{S})-\left(1-\frac{2}{3} \Phi^{4}\right) \frac{f-1}{\tau_{R}}-\frac{2}{3} \Phi^{4} \frac{f^{2}\left(f^{3}-1\right)}{3 \tau_{R}}$

Here, $\Phi$ is the effective polymer fraction defined in terms of the volume fraction of polymer in the solution $\varphi$ as well as the ratio between the entanglement molar mass of the PS melt $M_{\mathrm{em}}=13,300 \mathrm{~g} / \mathrm{mol}$ and the molar mass of the oligomeric styrene solvent $M_{\mathrm{os}}$,

$$
\begin{array}{ll}
\Phi=\frac{\varphi}{\varphi+(1-\varphi) \frac{M_{\mathrm{em}} / 4}{M_{\mathrm{os}}}} \quad \text { for } M_{\mathrm{os}}<M_{\mathrm{em}} / 4 \\
\Phi=\varphi \quad \text { for } M_{\mathrm{os}} \geq M_{\mathrm{em}} / 4
\end{array}
$$

As explained in Narimissa et al. (2020), the ratio $\Phi^{-2} /$ $\varphi^{-2}$ can be considered as a stretch enhancement factor, which increases the stretch potential of polystyrene dissolved in lower mass OS due to lower interchain pressure in these solutions. Again, in the limit of $f-1<<1$, the classical relation of Pearson et al. (1989), Eq. (15), is recovered from Eq. (29).

Predictions of Eqs. (13) and (29) were shown to be in excellent agreement with available elongational flow data of PS melts and solutions (Narimissa et al. 2020). In the following, we test the applicability of the new constitutive approach of Chapter 5 in modelling shear flow of polystyrene dissolved in $2 \mathrm{k}$ and $1 \mathrm{k}$ oligomeric styrene. However, a preliminary analysis revealed that the stretch enhancement due to the use of oligomeric solvents with $M_{\mathrm{os}}<M_{\mathrm{em}} / 4$, as predicted by Eq. (30), does not seem to occur in shear flow. We attribute this again to the rotational flow component of shear flow, which brings polymer chains into closer contact with each other, thereby increasing the interchain pressure and reducing the stretch potential. Therefore, for the $2 \mathrm{k}$ and $1 \mathrm{k}$ solutions considered here, we take $\Phi=\varphi$ in shear flow and propose the following stretch evolution equation for the PS solutions in conjunction with the stress tensor Eq. (24),

$\frac{\partial f(t, 0)}{\partial t}=f(t, 0)(\kappa: \mathbf{S}(t, 0))-\left(1-\frac{2}{3} \varphi^{4}\right) \frac{f(t, 0)-1}{\tau_{R}}-\frac{2}{3} \varphi^{4} \frac{f(t, 0)^{2}\left(f(t, 0)^{3}-1\right)}{3 \tau_{R}}$

A frame-indifferent formulation of Eqs. (24) and (31) is given in the Appendix.

Figures 11, 12 and 13 show the modelling of the shear behaviours of the solutions PS-285/2k-65, PS-285/2k-47 and PS-545/1k-52 through our proposed model (i.e. Eqs. (24) and (31)). We note that in all cases, the agreement of the shear stress growth component and the data can be considered as excellent, if at higher shear rates, the experimental time delay of reaching the prescribed shear rate is taken into account and the minimum in the shear stress 

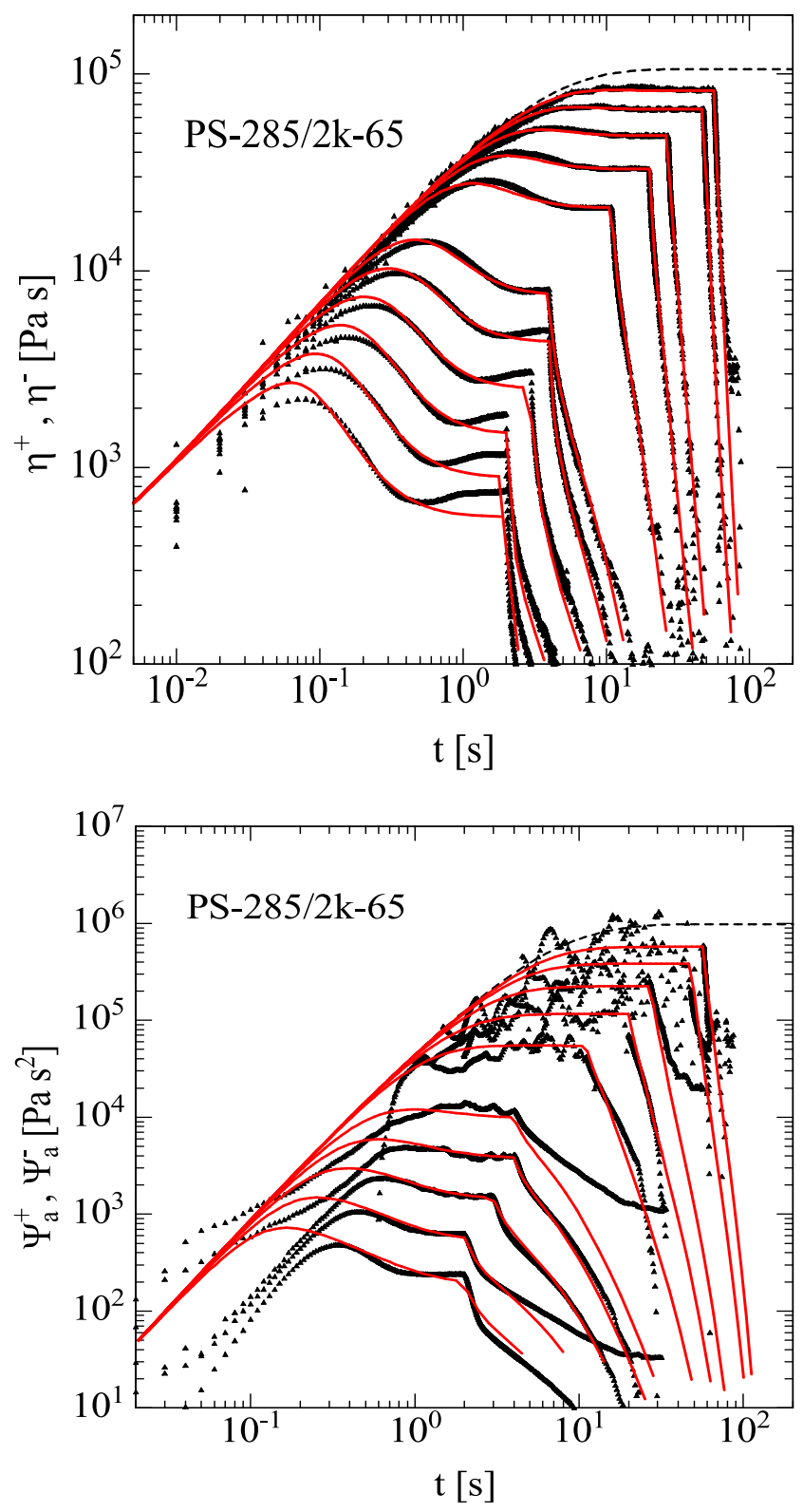

Fig. 11 Comparison between the predictions (red lines) of Eqs. (24) and (31) and the shear stress growth coefficient $\eta^{+}(t)$ and shear stress relaxation coefficient $\eta^{-}(t)$ as well as the apparent normal stress growth coefficient $\psi_{a}^{+}(t)$ and apparent normal stress relaxation coefficient $\psi_{a}^{-}(t)$ of PS-285/2k- 65 at $150{ }^{\circ} \mathrm{C}$. Shear rates from top to bottom: $0.1,0.178$, $0.316,0.562,1,1.78,3.16,5.62,10,17.8,31.6,56.2$ and $100 \mathrm{~s}^{-1}$ (only for shear stress growth coefficient)

followed by an increasing stress (i.e. the undershoot) is neglected. In particular, the location of the shear stress maximum is correctly predicted.

The growth coefficient of the apparent normal stress $\psi_{a}$ shows strong scatter at small shear rates due to low normal force signals, and with increasing shear rate, an increasing time delay relative to the predictions is observed due to limited rheometer compliance. Predictions of $\psi_{a}$ are in qualitative agreement with the data, and in the case
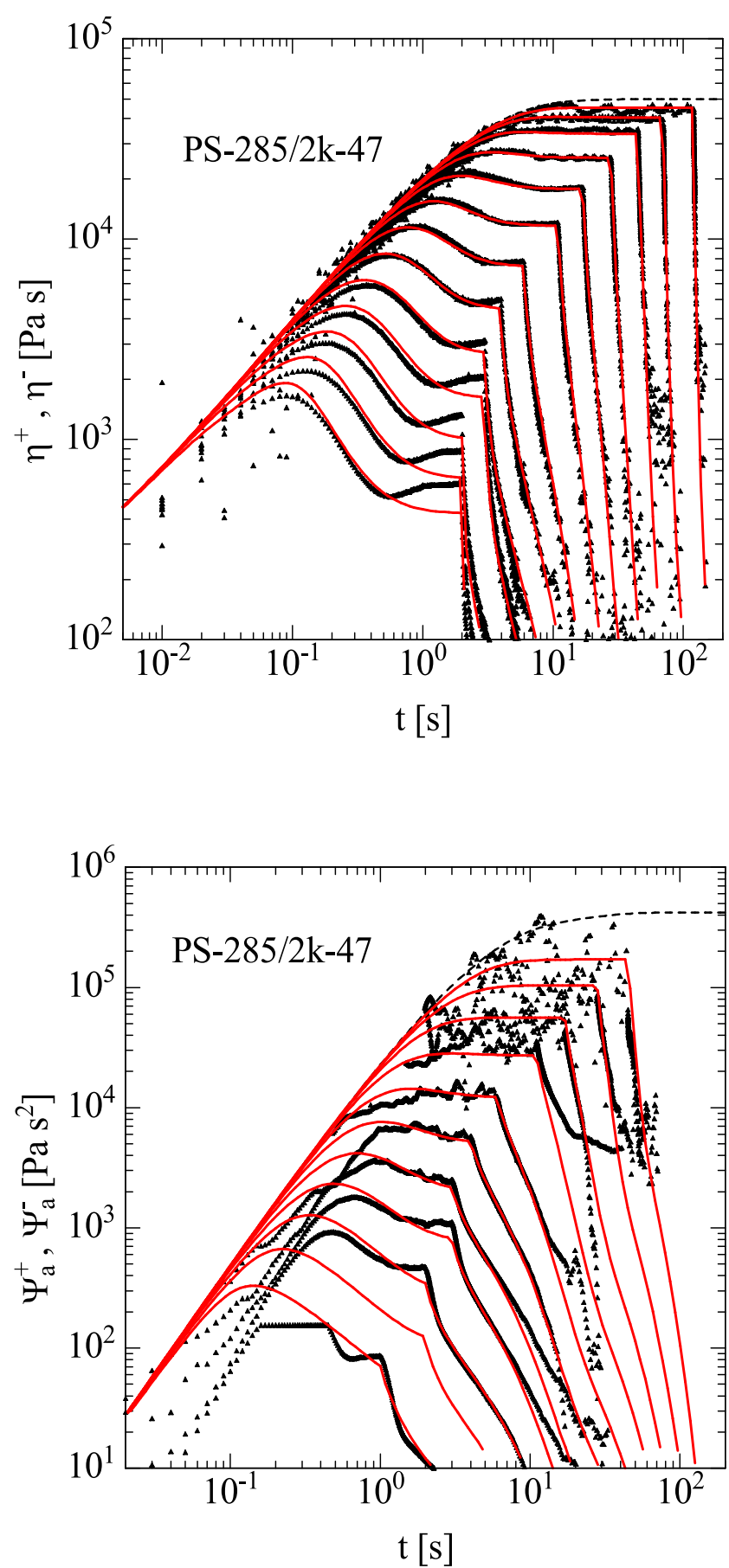

Fig. 12 Comparison between the predictions (red lines) of Eqs. (24) and (31) and the shear stress growth coefficient $\eta^{+}(t)$ and shear stress relaxation coefficient $\eta^{-}(t)$ as well as the apparent normal stress growth coefficient $\psi_{a}^{+}(t)$ and apparent normal stress relaxation coefficient $\psi_{a}^{-}(t)$ of PS-285/2k-47 at $140{ }^{\circ} \mathrm{C}$. Shear rates from top to bottom: $0.1,0.178$, $0.316,0.562,1,1.78,3.16,5.62,10,17.8,31.6,56.2$ and $100 \mathrm{~s}^{-1}$

of PS285k/2k-65 and PS285k/2k-47, they are even in quantitative agreement with the data when the steady state of the apparent normal stress coefficient is approached experimentally. The cut-off seen in the experimental data 

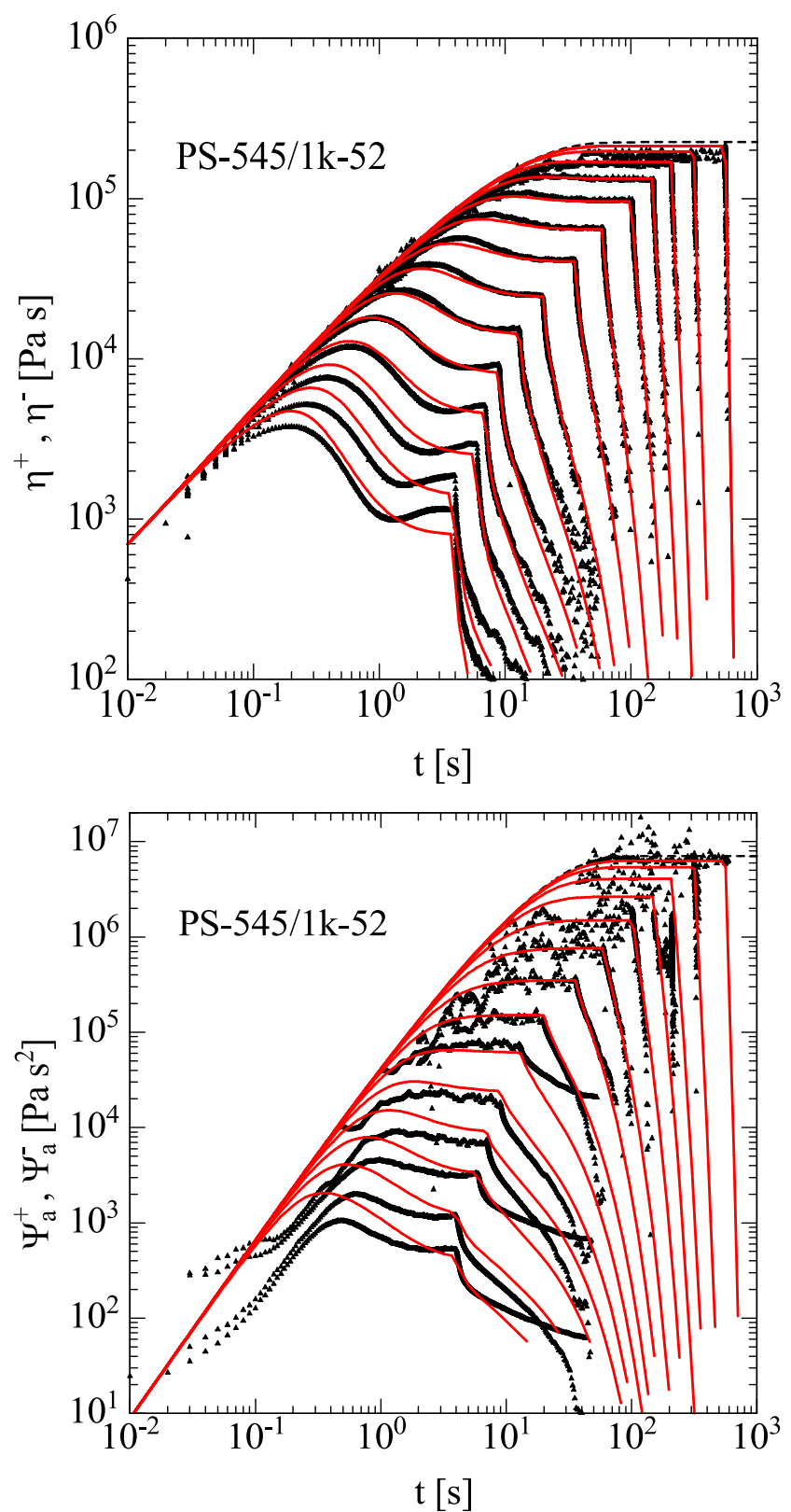

Fig. 13 Comparison between the predictions (red lines) of Eqs. (24) and (31) and the shear stress growth coefficient $\eta^{+}(t)$ and shear stress relaxation coefficient $\eta^{-}(t)$ as well as the apparent normal stress growth coefficient $\psi_{a}^{+}(t)$ and the apparent normal stress relaxation coefficient of PS-545/1k-52 at $130{ }^{\circ} \mathrm{C}$. Shear rates from top to bottom: 0.0178, 0.0316, $0.0562,0.1,0.178,0.316,0.562,1,1.78,3.16,5.62,10,17.8$ and $31.6 \mathrm{~s}^{-1}$

of $\psi_{a}$ at the highest shear rate in Fig. 12 is caused by the overload of the normal force transducer. For PS545k/1k52 , predictions indicate that at higher shear rates, a stronger than experimentally detected normal stress overshoot is expected, and the steady state is only reached at very high shear strains. Whether this discrepancy between model and data is caused by experimental issues or by limitations of the model is an open question, and further research in this area is required.

\section{Conclusions}

We have investigated the shear data of Schweizer et al. (2008) and Costanzo et al. (Costanzo et al. 2016, Costanzo et al. 2019) on nearly monodisperse polystyrene melts and solutions obtained by cone-partitioned-plate (CPP) shear rheometry. The extended interchain pressure (EIP) model, which allows a quantitative modelling of elongation flow of polymer melts and solutions, provides excellent prediction of the stress overshoot of both the stress growth coefficients of the shear stress, $\eta^{+}(t)$, and the first normal stress difference, $\psi_{1}^{+}(t)$, but overpredicts the steady-state shear viscosity and the steady-state first normal stress coefficient at shear rates $\dot{\gamma} \geq 10 s^{-1}$. The introduction of constraint release function $C R_{2}$ into the EIP model improves the predictions of the steady-state shear viscosity and the first normal stress; yet, it causes underprediction of the overshoots in $\eta^{+}(t)$ and $\psi_{1}^{+}(t)$ at all shear rates. At increasing values of the constraint release parameter $\beta$, this modelling approach (EIP with $C R_{2}$ ) converges to the Doi-Edwards model with independent alignment (DEIA) through the suppression of stretch evolution, i.e. $f_{i}\left(t, t^{\prime}\right) \cong 1$. The DEIA model shows excellent quantitative predictions of the steady-state shear viscosity and the first normal stress coefficient while it underpredicts the overshoot in $\eta^{+}(t)$ and does not predict an overshoot in $\psi_{1}^{+}(t)$. Consequently, we propose a novel constitutive model consisting of a combination of the DEIA and the EIP model. In this approach, we distinguish between entanglements created by reptation before the inception of flow at time $t=0$, i.e. at times $-\infty<t \leq 0$, and those created by reptation at time $t^{\prime}$ during flow, i.e. at times $0<t^{\prime} \leq t$. Entanglements created before inception of flow experience the stretch $f(t, 0)$ and the orientation $\mathbf{S}_{D E}^{I A}(t, 0)$, while due to the rotationality of shear flow, entanglements created after inception of flow are not stretched, i.e. $f\left(t, t^{\prime}\right)=1$, but are only oriented according to the relative orientation $\mathbf{S}_{D E}^{I A}\left(t, t^{\prime}\right)$ between times $t^{\prime}$ and $t$. Hence, the stress tensor of this novel constitutive approach is given as,

$\boldsymbol{\sigma}(t)=G(t) f^{2}(t, 0) \mathbf{S}_{D E}^{I A}(t, 0)+\int_{0}^{t} \frac{\partial G\left(t-t^{\prime}\right)}{\partial t^{\prime}} \mathbf{S}_{D E}^{I A}\left(t, t^{\prime}\right) d t^{\prime}$

with the stretch evolution equation for polymer melts

$\frac{\partial f(t, 0)}{\partial t}=f(t, 0)(\kappa: \mathbf{S}(t, 0))-\frac{1}{3} \frac{f(t, 0)-1}{\tau_{R}}-\frac{2}{3} \frac{f(t, 0)^{2}\left[f(t, 0)^{3}-1\right]}{3 \tau_{R}}$

We further showed that for the PS solutions in $2 \mathrm{k}$ and $1 \mathrm{k}$ oligomeric styrene considered in this study, the stretch evolution equation can be expressed as 
$\frac{\partial f(t, 0)}{\partial t}=f(t, 0)(\kappa: \mathbf{S}(t, 0))-\left(1-\frac{2}{3} \varphi^{4}\right) \frac{f(t, 0)-1}{\tau_{R}}-\frac{2}{3} \varphi^{4} \frac{f(t, 0)^{2}\left(f(t, 0)^{3}-1\right)}{3 \tau_{R}}$

For steady-state shear flow, the stress tensor (Eq. (24')) converges to the steady-state value of the DEIA model,

$\boldsymbol{\sigma}(\infty)=\int_{-\infty}^{+\infty} \frac{\partial G\left(t-t^{\prime}\right)}{\partial t^{\prime}} \mathbf{S}_{D E}^{I A}\left(t, t^{\prime}\right) d t^{\prime}$

Equations (24') and (25') for melts and Eq. (24') and (31') for solutions combine the description of the stress overshoot of the shear viscosity (resulting from a combination of stretch and orientation) as well as the stress overshoot of the first normal stress coefficient (which is an effect of the stretch alone), with the steady-state viscosity and first normal stress of the DEIA model. A frame-indifferent formulation of Eqs. (24') and (25') can be obtained by considering the "flow strength" or "rotationality" of deformation as shown in the Appendix.

Our proposed constitutive model achieves excellent agreement with the experimental data of the overshoot and the steady-state values of shear viscosity (if the observed stress minimum is taken as the steady state) and the first or apparent normal stress as well as the stress relaxation of PS melts (PS206k, PS-133k, PS-185k, PS-200k) and PS solutions (PS285/ 2k-65, PS285/2k-47 and PS545/1k-52) at a broad range of shear rates. The assumption of "non-stretching" of entanglements created during rotational flow is therefore in agreement with available experimental evidence. Future research may show whether this effect has a possible relationship to the concept of molecular tumbling. The model does not require any nonlinear-viscoelastic parameter, but relies solely on the linear-viscoelastic relaxation modulus $G(t)$ and the Rouse stretch relaxation time $\tau_{R}$, which in turn is determined by $G(t)$ and the molar mass of the polystyrene melt.

Acknowledgements We are greatly indebted to Salvatore Costanzo for sharing his data and for helpful suggestions and discussions.

Funding Information Open Access funding provided by Projekt DEAL.

\section{Appendix}

Thompson and Souza Mendez (Thompson and Souza Mendes 2005a; Thompson and Souza Mendes 2005b) following the earlier work of Astarita (1979) defined a persistence-ofstraining tensor $\mathbf{P}$

$$
\mathbf{P} \equiv \mathbf{D} \overline{\mathbf{W}}-\overline{\mathbf{W}} \mathbf{D}
$$

D is the rate of deformation tensor

$\mathbf{D}=\frac{1}{2}\left(\kappa+\kappa^{\mathbf{T}}\right)$

and $\overline{\mathbf{W}}$ the relative rate of rotation tensor

$\overline{\mathbf{W}}=\frac{1}{2}\left(\kappa-\kappa^{\mathbf{T}}\right)-\Omega=\mathbf{W}-\Omega$

where $\Omega$ is the tensor that gives the rate of rotation of the eigenvectors of $\mathbf{D}$. When $\mathbf{P}=0$, the flow is extensional corresponding to total persistence of straining.

We note in passing that the constraint release function $C R_{2}$ in Eq. (18) can be redefined in terms of the persistence-ofstraining tensor as

$C R_{2}=\frac{1}{2} \sqrt{2|\mathbf{P}: \mathbf{S}|}=\sqrt{|\overline{\mathbf{W}} \cdot \mathbf{D}: \mathbf{S}|}$

For shear flow, $\overline{\mathbf{W}}=\mathbf{W}$, and Eq. (18) is recovered from Eq. (35).

The intensity of $\mathbf{P}$ is a local measure of how far from maximum is the persistence of straining in a given flow. Thompson and Souza Mendez (Thompson and Souza Mendes 2005a, Thompson and Souza Mendes 2005b) define this intensity as

$P=\sqrt{\frac{1}{2} \mathbf{P}: \mathbf{P}}$

From Eq. (36) follows that $P=0$ for extensional flow, $P=$ $D: D$ for shear flow and $P>>D: D$ for flows which approach the limit of rigid body motion (Thompson and Souza Mendes 2005a, Thompson and Souza Mendes 2005b). Depending on the value of $P$, a frame-indifferent dimensionless measure of flow type can be constructed

$$
r(P)=1 \text { for } P=0
$$

$r(P)=1-\frac{P}{\mathbf{D}: \mathbf{D}}$ for $P \leq \mathbf{D}: \mathbf{D}$ and $\mathbf{D}: \mathbf{D}>0$

$r(P)=0$ for $P \geq \mathbf{D}: \mathbf{D}$ and $\mathbf{D}: \mathbf{D}>0$

Thus, $r(P)$ can be considered as a measure of "flow strength" (Tanner and Huilgol 1975), which decreases with increasing "rotationality" from $r=1$ (irrotational or extensional flow, also called "strong" flow) to $r=0$ for shear flow ("marginally weak" flow) and flows approaching the limit of rigid body motion ("weak" flows). Note that we consider "no flow", i.e. $P=0$ and simultaneously $\mathbf{D}: \mathbf{D}=0$, as irrotational and assign a value of $r=1$.

We can now make use of the rotationality parameter $r(P)$ to express stretch evolution Eq. (14) for the stretch $f\left(t, t^{\prime}\right)$ in a frame invariant way as 
$\frac{\partial f}{\partial t}=f\left[\kappa(t) r\left(t^{\prime}\right)\right]: \mathbf{S}\left(t, t^{\prime}\right)-\frac{1}{3} \frac{f-1}{\tau_{R}}-\frac{2}{3} \frac{f^{2}\left(f^{3}-1\right)}{3 \tau_{R}}$

with the starting condition $f\left(t=t^{\prime}, t^{\prime}\right)=1$.

For extensional flow, $r\left(t^{\prime}\right)=1$ for all times $t^{\prime}$, and Eq. (14) is recovered from Eq. (38).

For start-up of shear flow at time $t=0$ with $\kappa(t \leq 0)=0$ and $\kappa(t>0)=\kappa$, the rotationality parameter is given by $r\left(t^{\prime}>0\right)=$ 0. It then follows from Eq. (38) that

$f\left(t, t^{\prime}>0\right)=1$

because the first term on the right-hand side of Eq. (38) is zero for $t^{\prime}>0$, i.e. as intended, stretch of chain segments created during shear flow is fully suppressed. On the other hand, the evolution of stretch of entanglements existing before the startup of flow is given by,

$$
\begin{aligned}
& \frac{\partial f\left(t, t^{\prime} \leq 0\right)}{\partial t} \\
& =f\left(\kappa(t): \mathbf{S}\left(t, t^{\prime} \leq 0\right)\right)-\frac{1}{3} \frac{f-1}{\tau_{R}}-\frac{2}{3} \frac{f^{2}\left[f^{3}-1\right]}{3 \tau_{R}}
\end{aligned}
$$

as $\kappa\left(t^{\prime} \leq 0\right)=0$ and therefore according to Eq. (37a) $r\left(t^{\prime} \leq\right.$ $0)=1$. Equation (40) is equivalent to Eq. (25). From Eqs. (7) and (39), the extra stress tensor for start-up of shear flow is then given by

$\boldsymbol{\sigma}(t)=G(t) f^{2}(t, 0) \mathbf{S}_{D E}^{I A}(t, 0)+\int_{0}^{t} \frac{\partial G\left(t-t^{\prime}\right)}{\partial t^{\prime}} \mathbf{S}_{D E}^{I A}\left(t, t^{\prime}\right) d t^{\prime}$

which is identical to Eq. (24).

Similarly, in the case of PS solutions in oligomeric styrene, the stretch evolution Eq. (29) can be expressed in a frame invariant way as

$$
\begin{aligned}
\frac{\partial f}{\partial t} & =f\left[\kappa(t) r\left(t^{\prime}\right)\right] \\
& : \mathbf{S}\left(t, t^{\prime}\right)-\left(1-\frac{2}{3} \Theta^{4}\right) \frac{f-1}{\tau_{R}}-\frac{2}{3} \Theta^{4} \frac{f^{2}\left(f^{3}-1\right)}{3 \tau_{R}}
\end{aligned}
$$

with effective polymer fraction $\Theta$

$\Theta=\varphi+(\Phi-\varphi) r(t)$

and $\Phi$ given by Eq. (30).

For extensional flow, $r(t)=1$ and $r\left(t^{\prime}\right)=1$ for all times $t$ and $t^{\prime}$, and Eq. (29) is recovered from Eq. (42).

For start-up of shear flow at time $t=0$ and $r\left(t^{\prime}>0\right)=0$, and therefore

$f\left(t, t^{\prime}>0\right)=1$
Stretch of chain segments created during shear flow is fully suppressed in solutions as in melts. For entanglements existing before the start-up of flow, the effective polymer volume fraction reduces for $t>0$ and $r(t>0)=0$ to $\Theta=\varphi+(\Phi$ $-\varphi) r(t)=\varphi$, and as $\kappa\left(t^{\prime} \leq 0\right)=0$ and therefore according to Eq. (37a) $r\left(t^{\prime} \leq 0\right)=1$, the evolution of the stretch is given by

$\frac{\partial f}{\partial t}=f\left(\kappa(t): \mathbf{S}\left(t, t^{\prime} \leq 0\right)\right)-\left(1-\frac{2}{3} \varphi^{4}\right) \frac{f-1}{\tau_{R}}-\frac{2}{3} \varphi^{4} \frac{f^{2}\left(f^{3}-1\right)}{3 \tau_{R}}$

Eq. (45) is equivalent to Eq. (29).

Open Access This article is licensed under a Creative Commons Attribution 4.0 International License, which permits use, sharing, adaptation, distribution and reproduction in any medium or format, as long as you give appropriate credit to the original author(s) and the source, provide a link to the Creative Commons licence, and indicate if changes were made. The images or other third party material in this article are included in the article's Creative Commons licence, unless indicated otherwise in a credit line to the material. If material is not included in the article's Creative Commons licence and your intended use is not permitted by statutory regulation or exceeds the permitted use, you will need to obtain permission directly from the copyright holder. To view a copy of this licence, visit http://creativecommons.org/licenses/by/4.0/.

\section{References}

Acharya M, Bhattacharjee P, Nguyen D, Sridhar T (2008) Are entangled polymeric solutions different from melts? AIP Conference Proceedings, vol 1027. AIP, pp 391-393

Anwar M, Graham RS (2019) Nonlinear shear of entangled polymers from nonequilibrium molecular dynamics. Journal of Polymer Science Part B, Polymer Physics

Astarita G (1979) Objective and generally applicable criteria for flow classification. J Non-Newtonian Fluid Mech 6:69-76

Auhl D, Ramirez J, Likhtman AE, Chambon P, Fernyhough C (2008) Linear and nonlinear shear flow behavior of monodisperse polyisoprene melts with a large range of molecular weights. J Rheol 52: 801-835. https://doi.org/10.1122/1.2890780

Auhl D, Chambon P, McLeish TCB, Read DJ (2009) Elongational flow of blends of long and short polymers: effective stretch relaxation time. Phys Rev Letters 103

Bach A, Almdal K, Rasmussen HK, Hassager O (2003) Elongational viscosity of narrow molar mass distribution polystyrene. Macromolecules 36:5174-5179

Bastian H (2001) Non-linear viscoelasticity of linear and long-chainbranched polymer melts in shear and extensional flows Institut für Kunststofftechnik, vol PhD. Universität Stuttgart, Stuttgart, pp 130

Bernstein B, Kearsley E, Zapas L (1963) A study of stress relaxation with finite strain. Transactions of The Society of Rheology (1957-1977) 7:391-410

Bhattacharjee PK, Oberhauser JP, McKinley GH, Leal LG, Sridhar T (2002) Extensional rheometry of entangled solutions. Macromolecules 35: 10131-10148. DOI Export Date 14 September 2013

Boukany PE, Wang S-Q (2007) A correlation between velocity profile and molecular weight distribution in sheared entangled polymer solutions. J Rheol 51:217-233

Costanzo S (2020) Private communication with Salvatore Costanzo

Costanzo S, Huang Q, Ianniruberto G, Marrucci G, Hassager O, Vlassopoulos D (2016) Shear and extensional rheology of 
polystyrene melts and solutions with the same number of entanglements. Macromolecules 49:3925-3935

Costanzo S, Ianniruberto G, Marrucci G, Vlassopoulos D (2018) Measuring and assessing first and second normal stress differences of polymeric fluids with a modular cone-partitioned plate geometry. Rheol Acta 57:363-376

Costanzo S, Pasquino R, Läuger J, Grizzuti N (2019) Milligram size rheology of molten polymers. Fluids 4:28

Daoud M, De Gennes P (1979) Some remarks on the dynamics of polymer melts. J Poly Sci: Polymer Physics Edition 17:1971-1981

De Gennes PG (1971) Reptation of a polymer chain in the presence of fixed obstacles. J Chem Phys 55:572-579

Dealy JM, Read DJ, Larson RG (2018) Structure and rheology of molten polymers: from structure to flow behavior and back again. Carl Hanser Verlag GmbH Co KG

Doi M (1981) Explanation for the 3.4 power law of viscosity of polymeric liquids on the basis of the tube model. J Poly Sci: Polymer Letters Edition 19:265-273

Doi M, Edwards SF (1978a) Dynamics of concentrated polymer systems. Part 2. - molecular motion under flow. J Chem Soc, Faraday Transactions 2: Molecular and Chemical Physics 74: 1802-1817. DOI https://doi.org/10.1039/F29787401802

Doi M, Edwards SF (1978b) Dynamics of concentrated polymer systems. Part 3. - the constitutive equation. J Chem Soc, Faraday Transactions 2: Molecular and Chemical Physics 74:1818-1832

Doi M, Edwards SF (1979) Dynamics of concentrated polymer systems. Part 4. - rheological properties. J Chem Society, Faraday Transactions 2: Molecular and Chemical Physics 75:38-54 DOI Export Date 24 August 2013

Doi M, Edwards SF (1986) The theory of polymer dynamics. Oxford University Press, Oxford

Ferry JD (1980) Viscoelastic properties of polymers. John Wiley and Sons, New York

Fukuda M, Osaki K, Kurata M (1975) Nonlinear viscoelasticity of polystyrene solutions. I. Strain-dependent relaxation modulus. Journal of Polymer Science: Polymer Physics Edition 13:1563-1576

Huang Q, Alvarez NJ, Matsumiya Y, Rasmussen HK, Watanabe H, Hassager O (2013a) Extensional rheology of entangled polystyrene solutions suggests importance of nematic interactions. ACS Macro Lett 2:741-744

Huang Q, Mednova O, Rasmussen HK, Alvarez NJ, Skov AL, Almdal K, Hassager O (2013b) Concentrated polymer solutions are different from melts: role of entanglement molecular weight. Macromolecules 46:5026-5035

Huang Q, Hengeller L, Alvarez NJ, Hassager O (2015) Bridging the gap between polymer melts and solutions in extensional rheology. Macromolecules 48:4158-4163

Ianniruberto G (2015) Extensional flows of solutions of entangled polymers confirm reduction of friction coefficient. Macromolecules 48: 6306-6312

Ianniruberto G, Marrucci G (1996) On compatibility of the cox-Merz rule with the model of Doi and Edwards. J Non-Newtonian Fluid Mech 65:241-246. https://doi.org/10.1016/0377-0257(96)01433-4

Isaki T, Takahashi M, Urakawa O (2003) Biaxial damping function of entangled monodisperse polystyrene melts: comparison with the Mead-Larson-Doi model. J Rheol 47:1201-1210 DOI Export Date 24 August 2013

Keentok M, Xue S-C (1999) Edge fracture in cone-plate and parallel plate flows. Rheol Acta 38:321-348

Larson R (2001) Combinatorial rheology of branched polymer melts. Macromolecules 34:4556-4571

Laun HM, Schuch H (1989) Transient elongational viscosities and drawability of polymer melts. J Rheol 33:119-175. https://doi.org/ $10.1122 / 1.550058$

Luap C, Müller C, Schweizer T, Venerus DC (2005) Simultaneous stress and birefringence measurements during uniaxial elongation of polystyrene melts with narrow molecular weight distribution. Rheol Acta 45:83-91

Marrucci G (1996) Dynamics of entanglements: a nonlinear model consistent with the Cox-Merz rule. J Non-Newtonian Fluid Mech 62: 279-289 DOI Export Date 62(24 August 2013):279-289

Marrucci G, de Cindio B (1980) The stress relaxation of molten PMMA at large deformations and its theoretical interpretation. Rheol Acta 19:68-75. https://doi.org/10.1007/BF01523856

Marrucci G, Grizzuti N (1988) Fast flows of concentrated polymers: predictions of the tube model on chain stretching. Gazz Chim Itali 118:179-185

Marrucci G, Ianniruberto G (2004) Interchain pressure effect in extensional flows of entangled polymer melts. Macromolecules 37:3934-3942

Masubuchi Y, Ianniruberto G, Marrucci G (2018) Stress undershoot of entangled polymers under fast startup shear flows in primitive chain network simulations. Nihon Reoroji Gakkaishi 46:23-28

McLeish TCB, Larson RG (1998) Molecular constitutive equations for a class of branched polymers: the pom-pom polymer. J Rheol 42:81110. https://doi.org/10.1122/1.550933

Mead DW, Yavich D, Leal LG (1995) The reptation model with segmental stretch - II. Steady flow properties Rheol Acta 34: 360-383 DOI Export Date (24 August 2013)

Meissner J (1972) Modifications of the Weissenberg rheogoniometer for measurement of transient rheological properties of molten polyethylene under shear. Comparison with tensile data J Appl Polym Sci 16:2877-2899

Meissner J, Garbella R, Hostettler J (1989) Measuring normal stress differences in polymer melt shear flow. J Rheol 33:843-864

Menezes E, Graessley W (1982) Nonlinear rheological behavior of polymer systems for several shear-flow histories. J Poly Sci Part B: Poly Phys 20:1817-1833

Moorcroft RL, Fielding SM (2013) Criteria for shear banding in timedependent flows of complex fluids. Phys Rev Lett 110:086001

Nafar Sefiddashti MN, Edwards B, Khomami B (2014) Individual chain dynamics of a polyethylene melt undergoing steady shear flow. J Rheol 59(1):119-153

Nafar Sefiddashti MH, Edwards B, Khomami B (2015) Individual chain dynamics of a polyethylene melt undergoing steady shear flow. J Rheol 59:119-153

Nafar Sefiddashti MH, Edwards BJ, Khomami B (2017) Evaluation of reptation-based modeling of entangled polymeric fluids including chain rotation via nonequilibrium molecular dynamics simulation. Physical Review Fluids 2:083301

Nafar Sefiddashti MH, Edwards BJ, Khomami B (2019a) Elucidating the molecular rheology of entangled polymeric fluids via comparison of atomistic simulations and model predictions. Macromolecules 52: 8124-8143

Nafar Sefiddashti MH, Edwards BJ, Khomami B (2019b) Individual molecular dynamics of an entangled polyethylene melt undergoing steady shear flow: steady-state and transient dynamics. Polymers 11:476

Narimissa E, Wagner MH (2016) A hierarchical multi-mode MSF model for long-chain branched polymer melts part III: shear flow. Rheol Acta 55:633-639. https://doi.org/10.1007/s00397-016-0939-2

Narimissa E, Wagner MH (2016a) From linear viscoelasticity to elongational flow of polydisperse polymer melts: the hierarchical multi-mode molecular stress function model. Polymer 104:204 214. https://doi.org/10.1016/j.polymer.2016.06.005

Narimissa E, Wagner MH (2016b) A hierarchical multi-mode molecular stress function model for linear polymer melts in extensional flows. J Rheol 60:625-636. https://doi.org/10.1122/1.4953442

Narimissa E, Wagner MH (2018) Review of the hierarchical multi-mode molecular stress function model for broadly distributed linear and LCB polymer melts. Poly Eng Sci 59:573-583. https://doi.org/10. 1002/pen.24972 
Narimissa E, Wagner MH (2019) Review on tube model based constitutive equations for polydisperse linear and long-chain branched polymer melts. J Rheol 63:361-375. https://doi.org/10.1122/1.5064642

Narimissa E, Rolón-Garrido VH, Wagner MH (2015) A hierarchical multi-mode MSF model for long-chain branched polymer melts part I: elongational flow. Rheol Acta 54:779-791. https://doi.org/10. 1007/s00397-015-0879-2

Narimissa E, Rolón-Garrido VH, Wagner MH (2016) A hierarchical multi-mode MSF model for long-chain branched polymer melts part II: multiaxial extensional flows. Rheol Acta 55:327-333. https://doi. org/10.1007/s00397-016-0922-y

Narimissa E, Huang Q, Wagner MH (2020) Elongational rheology of polystyrene melts and solutions: concentration dependence of the interchain tube pressure effect. J Rheol 64:95-110. https://doi.org/ $10.1122 / 1.5100671$

Nielsen JK, Rasmussen HK, Hassager O (2008) Stress relaxation of narrow molar mass distribution polystyrene following uniaxial extension. J Rheol 52:885-899

O'Connor TC, Hopkins A, Robbins MO (2019) Stress relaxation in highly oriented melts of entangled polymers. Macromolecules 52:8540 8550. https://doi.org/10.1021/acs.macromol.9b01161

Osaki K, Fukuda M, Ohta SI, Kim BS, Kurata M (1975) Nonlinear viscoelasticity of polystyrene solutions. II. Non-Newtonian viscosity. Journal of Polymer Science: Polymer Physics Edition 13:1577-1589

Osaki K, Ohta S, Fukuda M, Kurata M (1976) Nonlinear viscoelasticity of polystyrene solutions. III. Stress development at the start of steady shear flow and an experimental check of some constitutive models. Journal of Polymer Science: Polymer Physics Edition 14:1701-1715

Osaki K, Nishizawa K, Kurata M (1982) Material time constant characterizing the nonlinear viscoelasticity of entangled polymeric systems. Macromolecules 15:1068-1071 DOI Export Date 24 August 2013

Osaki K, Inoue T, Isomura T (2000) Stress overshoot of polymer solutions at high rates of shear. J Polym Sci B Polym Phys 38:1917-1925

Park GW, Ianniruberto G (2017) Flow-induced nematic interaction and friction reduction successfully describe ps melt and solution data in extension startup and relaxation. Macromolecules 50:4787-4796

Park SJ, Larson RG (2003) Dilution exponent in the dynamic dilution theory for polymer melts. J Rheol 47:199-211

Pearson DS, Kiss AD, Fetters LJ, Doi M (1989) Flow-induced birefringence of concentrated polyisoprene solutions. J Rheol 33:517-535. https://doi.org/10.1122/1.550026

Ravindranath S, Wang S-Q, Olechnowicz M, Quirk RP (2008) Banding in simple steady shear of entangled polymer solutions. Macromolecules 41:2663-2670

Samurkas T, Dealy J, Larson R (1989) Strong extensional and shearing flows of a branched polyethylene. J Rheol 33:559-578

Schweizer T, Schmidheiny W (2013) A cone-partitioned plate rheometer cell with three partitions (CPP3) to determine shear stress and both normal stress differences for small quantities of polymeric fluids. J Rheol 57:841-856

Schweizer T, Stöckli M (2008) Departure from linear velocity profile at the surface of polystyrene melts during shear in cone-plate geometry. J Rheol 52:713-727

Schweizer T, van Meerveld J, Öttinger HC (2004) Nonlinear shear rheology of polystyrene melt with narrow molecular weight distribution-experiment and theory. J Rheol 48:1345-1363

Schweizer T, Hostettler J, Mettler F (2008) A shear rheometer for measuring shear stress and both normal stress differences in polymer melts simultaneously: the MTR 25. Rheol Acta 47:943-957

Smith DE, Babcock HP, Chu S (1999) Single-polymer dynamics in steady shear flow. Science 283:1724-1727

Snijkers F, Vlassopoulos D (2011) Cone-partitioned-plate geometry for the ARES rheometer with temperature control. J Rheol 55:1167-1186
Stephanou PS, Schweizer T, Kröger M (2017) Communication: appearance of undershoots in start-up shear: experimental findings captured by tumbling-snake dynamics. J Chem Phys 146:161101

Takahashi M, Isaki T, Takigawa T, Masuda T (1993) Measurement of biaxial and uniaxial extensional flow behavior of polymer melts at constant strain rates. J Rheol 37:827-846. https://doi.org/10.1122/1.550397

Tanner R, Huilgol R (1975) On a classification scheme for flow fields. Rheol Acta 14:959-962

Tanner R, Keentok M (1983) Shear fracture in cone-plate rheometry. J Rheol 27:47-57

Tapadia P, Wang S-Q (2006) Direct visualization of continuous simple shear in non-Newtonian polymeric fluids. Phys Rev Lett 96:016001

Thompson RL, Souza Mendes PR (2005a) Considerations on kinematic flow classification criteria. J Non-Newtonian Fluid Mech 128:109-115

Thompson RL, Souza Mendes PR (2005b) Persistence of straining and flow classification. Int J Eng Sci 43:79-105

Venerus DC (2007) Free surface effects on normal stress measurements in cone and plate flow. Applied rheology 17: 36494-36491-36494-36496

Wagner M (1977) Prediction of primary normal stress difference from shear viscosity data using a single integral constitutive equation. Rheol Acta 16:43-50

Wagner MH (2014) Scaling relations for elongational flow of polystyrene melts and concentrated solutions of polystyrene in oligomeric styrene. Rheol Acta 53:765-777. https://doi.org/10.1007/s00397-014-0791-1

Wagner MH, Rolón-Garrido VH (2009a) Nonlinear rheology of linear polymer melts: modeling chain stretch by interchain tube pressure and Rouse time. Korea Australia Rheol J 21:203-211

Wagner MH, Rolón-Garrido VH (2009b) Recent advances in constitutive modeling of polymer melts. Novel trends of rheology III. AIP Conf Proc 1152:16-31. https://doi.org/10.1063/1.3203266

Wagner MH, Rolón-Garrido VH (2010) The interchain pressure effect in shear rheology. Rheol Acta 49:459-471

Wagner M, Schaeffer J (1992a) Nonlinear strain measures for general biaxial extension of polymer melts. J Rheol 36:1-26

Wagner MH, Schaeffer J (1992b) Nonlinear strain measures for general biaxial extension of polymer melts. J Rheol 36:1-26 DOI Export Date 24 August 2013

Wagner MH, Schaeffer J (1993) Rubbers and polymer melts: universal aspects of nonlinear stress-strain relations. J Rheol 37:643-661

Wagner MH, Schaeffer J (1994) Assessment of nonlinear strain measures for extensional and shearing flows of polymer melts. Rheol Acta 33: 506-516 DOI Export Date 24 August 2013

Wagner MH, Rubio P, Bastian H (2001) The molecular stress function model for polydisperse polymer melts with dissipative convective constraint release. J Rheol 45:1387-1412. https://doi.org/10.1122/1.1413503

Wagner MH, Yamaguchi M, Takahashi M (2003) Quantitative assessment of strain hardening of low-density polyethylene melts by the molecular stress function model. J Rheol 47:779-793. https://doi. org/10.1122/1.1562155

Wagner MH, Kheirandish S, Hassager O (2005) Quantitative prediction of transient and steady-state elongational viscosity of nearly monodisperse polystyrene melts. J Rheol 49:1317-1327. https://doi.org/ 10.1122/1.2048741

Watanabe H, Ishida S, Matsumiya Y, Inoue T (2004) Test of full and partial tube dilation pictures in entangled blends of linear polyisoprenes. Macromolecules 37:6619-6631

Winter HH, Mours M (2006) The cyber infrastructure initiative for rheology. Rheol Acta 45:331-338

Publisher's note Springer Nature remains neutral with regard to jurisdictional claims in published maps and institutional affiliations. 